\title{
1 HNF4 factors control chromatin accessibility and are redundantly required for maturation of the fetal intestine
}

\author{
3 Lei Chen ${ }^{1,2}$, Natalie H. Toke ${ }^{1}$, Shirley Luo ${ }^{1}$, Roshan P. Vasoya ${ }^{1}$, Rohit Aita ${ }^{1}$, Aditya Parthasarathy ${ }^{1}$, \\ 4 Yu-Hwai Tsai ${ }^{3}$, Jason R. Spence ${ }^{3,4,5,6}$, Michael P. Verzi ${ }^{1,2, *}$ \\ $5{ }^{1}$ Department of Genetics, Human Genetics Institute of New Jersey, Rutgers University, \\ 6 Piscataway, NJ 08854, USA \\ $7 \quad{ }^{2}$ Rutgers Cancer Institute of New Jersey, New Brunswick, NJ 08903, USA \\ $8{ }^{3}$ Department of Internal Medicine, Gastroenterology, University of Michigan Medical School, \\ 9 Ann Arbor, MI 48109, USA \\ $10{ }^{4}$ Department of Cell and Developmental Biology, University of Michigan Medical School, Ann \\ 11 Arbor, MI 48109, USA \\ 12 Department of Biomedical Engineering, University of Michigan College of Engineering, Ann \\ 13 Arbor, MI, USA. \\ $14{ }^{6}$ Center for Organogenesis, University of Michigan Medical School, Ann Arbor, MI 48109, USA \\ *Corresponding Author: verzi@biology.rutgers.edu (M.P.V.)
}

\section{ABSTRACT}

As an embryo matures into a fetus, cells undergo remarkable transitions, accompanied by shifts in transcription factor regulatory networks and chromatin landscapes. The mechanisms of these developmental transitions are not completely understood. The embryonic intestine transitions from a rapidly proliferating tube with pseudostratified epithelium prior to embryonic day (E) 14.5 , to an exquisitely folded columnar epithelium in the fetus. We sought to define factors that drive fetal maturation of the intestine. ATAC-seq profiling revealed a dramatic restructuring of intestinal chromatin during the embryonic-to-fetal transition, with CDX2 transcription factor motifs abundant at chromatin-accessible regions of the embryo, and hepatocyte nuclear factor 4 (HNF4) transcription factor motifs the most abundant in the fetal stages. Genetic inactivation of $H n f 4 \alpha$ and its paralog, $H n f 4 \gamma$, revealed that HNF4 factors are redundantly and vitally required for fetal maturation. In the embryo, CDX2 binds to and activates Hnf4 gene loci to drive HNF4 expression at fetal stages. HNF4 and CDX2 transcription factors then occupy shared genomic regulatory sites and are required for chromatin accessibility at genes expressed in the maturing fetal intestine. Thus, intestinal transcription factor regulatory networks shift to accompany changing chromatin landscapes and gene expression profiles that occur during the transition of an embryonic tissue to its mature state. 


\section{INTRODUCTION}

41 The developing embryo is a collection of partially-fated cells that expand exponentially during the stages of organogenesis, approximately E9.5 to E13.5 in mice (Cao et al., 2019). As development proceeds into fetal stages, specified cells undergo transitions to acquire characteristics of mature tissues. The mechanisms of these developmental transitions are not completely understood, but are of great importance in understanding the basic mechanisms of development and developmental disorders, and in facilitating efforts of regenerative medicine.

The embryonic gut tube arises from endoderm after gastrulation and is specified along the anterior-posterior axis into distinct derivative endodermal organs. The primitive gut divides into foregut, midgut and hindgut; the small intestine develops primarily from the midgut. Murine gut tube formation is completed by E9.5, and its inner lining consists of a highly-proliferative, pseudostratified epithelium as the gut tube elongates, with the most rapid growth occurring by E14.5. At E14.5 to E15.5, the tissue undergoes a remarkable transition to a columnar epithelium and the process of villus formation, elongation, and maturation occurs at E15.5 to E18.5 (Chin et al., 2017; Walton et al., 2016a; Wells and Spence, 2014). The mechanisms triggering this embryonic to fetal transition in the developing gut remain unclear.

A shift in transcription factor regulatory networks accompanies the majority of known cellular transitions (Niwa, 2018; Wilkinson et al., 2017). Transcription factors function at distal genomic regulatory regions known as enhancers, which are the primary drivers of tissue-specific gene expression (Consortium, 2012; Nord et al., 2013; Shen et al., 2012; Visel et al., 2009). In our efforts to understand mechanisms driving developmental transitions in the gut, we recently mapped chromatin profiles of esophagus, forestomach, hindstomach and small intestine over developmental time. We noted a clear transition in chromatin accessibility within the developing intestine that corresponds to the stage in which the morphogenetic events reshaping the intestine occur ( $\sim$ E15.5). The transcription factor, CDX2, operates on both sides of this developmental transition. In the early embryonic intestine (prior to E13.5), CDX2 is required for intestinal specification, and loss of CDX2 leads to ectopic features of stomach and esophageal tissues in the intestine (Banerjee et al., 2018; Gao et al., 2009; Grainger et al., 2010; Kumar et al., 2019). Acute inactivation of CDX2 at later developmental timepoints (post-E13.5) compromises mature tissue functions, but intestinal identity is maintained (Banerjee et al., 2018; Kumar et al., 2019; Verzi et al., 2011). While CDX2 is a clear driver of intestinal specification and plays an additional role in driving fetal maturation, it remains unclear how this developmental transition occurs.

Here, we aim to find transcription factors that could function specifically in intestinal maturation. We find that genomic regions that become increasingly accessible at fetal stages are most enriched for DNA-binding motifs known to bind to the HNF4 transcription factor family. In adult intestine, HNF4A and HNF4G have been shown to drive expression of genes important for digestive physiology (Boyd et al., 2009; Cattin et al., 2009; Lindeboom et al., 2018). HNF4A binding activity is modulated by the microbiome (Davison et al., 2017) and suppressed in a mouse model of ulcerative colitis (Chahar et al., 2014). We recently demonstrated that HNF4A 
while HNF4 transcription factors have been studied in a number of developmental contexts, their redundant functions have not been assayed in the context of the developing gut.

Hnf $4 \alpha$ deletion is embryonically lethal, with defects in visceral endoderm (VE) (Chen et al., 1994; Duncan et al., 1997). Complementation of $\mathrm{Hnf4} \mathrm{\alpha}^{-/-}$embryos with $\mathrm{Hnf} 4 \mathrm{\alpha}^{+/+}$VE by tetraploid aggregation (Duncan et al., 1997; Li et al., 2000) or conditional deletion of $H n f 4 \alpha$ (Babeu et al., 2009; Garrison et al., 2006; Parviz et al., 2003) allowed for the investigation of HNF4 function in later developmental stages. In the liver, $H n f 4 \alpha$ was dispensable for liver specification but was essential for hepatocyte differentiation (Hayhurst et al., 2001; Li et al., 2000; Parviz et al., 2003). However, no consequence of HNF4A loss in the developing small intestinal epithelium has been observed. This may be due to an inefficient depletion in the small intestine due to the mosaic action of the Foxa3-Cre driver, as suggested by Garrison et al. (Garrison et al., 2006). Inactivation of conditional Hnf4 $\alpha$ alleles using the Villin-Cre driver resulted in no embryonic phenotype (Babeu et al., 2009), possibly due to the relatively late onset of Villin-Cre expression (Madison et al., 2002) or unappreciated genetic redundancy with $H n f 4 \gamma$. Here, we demonstrate that intestinal expression of the Hnf4 paralogs is induced during the embryonic to fetal transition, and that Hnf4 genes depend upon CDX2 for expression in both mice and humans. Analysis of chromatin accessibility and ChIP-seq data in the developing and adult gut supports a model in which the transcription factor CDX2 activates Hnf4 gene expression, and together HNF4 and CDX2 drive tissue maturation by activating genes important for intestinal function in post-natal life. Finally, we generate a mouse model lacking HNF4 factors in the embryonic intestine to show that while HNF4 factors are dispensable for intestinal specification and villus morphogenesis, they are critical for maturation of the fetal intestine.

\section{RESULTS}

\section{Chromatin landscapes indicate that HNF4 factors are more likely to function in intestinal maturation than specification}

Deciphering the mechanisms of tissue maturation is a critical goal in understanding developmental disorders and facilitating efforts in regenerative medicine. Developmental transitions are accompanied by changes in chromatin accessibility and transcription factor regulatory networks. To better understand the regulatory mechanisms driving intestinal maturation, we re-analyzed ATAC-seq data (Banerjee et al., 2018) to identify chromatinaccessible regions in isolated intestinal epithelial cells (Fig.1A). Three categories of accessible regions were identified (MACS $P$ value $\leq 10^{-5}$ ). 2,644 regions (cluster 1 ) were accessible at E11.5 and remained accessible at all stages examined. 10,544 regions (cluster 2, "maturation enriched regions") of intestinal chromatin are progressively accessible, whereas 30,702 regions (cluster 3, "embryo enriched regions") lose accessibility from E11.5 to adult (Fig.1A, Table S1). Notably, a gain in accessibility at "maturation enriched regions" coincides with the loss of accessibility at "embryo enriched regions". This E14.5-16.5 stage is marked by a transition of the intestinal epithelium from a pseudostratified to columnar tissue. To identify the regulatory complexes likely operating during this developmental transition, we applied DNA-binding motif analysis. Regions selectively accessible in the maturing gut exhibit HNF4A/G as the top-scoring motif (Fig.1B, Table S1), suggesting that HNF4 factors function in maturation of the developing gut. 
Conversely, CDX2 is the most prevalent transcription factor motif in the 30,702 regions that are more accessible in embryonic epithelium (Fig.1B, Table S1). H3K27ac (Kazakevych et al., 2017), which marks active enhancer regions, is enriched at stage-specific accessible chromatin regions defined by ATAC-seq (Fig.1C), corroborating putative regulatory function of these regions. Gene ontology analysis suggests that genes nearby the accessible regions of the early embryonic intestine function in intestinal specification and morphogenesis, whereas genes nearby accessible chromatin in the maturing intestine exhibit function consistent with the mature tissue (Fig.1D, Table S1). DNA-binding motif enrichment analysis at each timepoint revealed that while CDX2 binding motifs are enriched as early as E11.5, HNF4 binding motifs are first detected at E14.5 and become more prevalent at accessible chromatin regions of fetal and adult stages (Fig.S1A). Mirroring their motif enrichment profiles, we find that $C d \times 2$ transcripts are highly and equally expressed across early embryonic to adult stages, whereas Hnf4 transcripts are not robustly expressed until fetal and adult stages (Fig.1E). Protein expression levels of HNF4 factors (Fig.S1B,C) appear to increase over developmental time, consistent with the increase in transcript levels. Similarly, human CDX2 expression is observed upon intestinal specification of hESC-derived endoderm by FGF and WNT, whereas expression of HNF4A and HNF4G is not observed until cultures are further matured into human intestinal organoids (Fig.1F, Fig.S1D). Taken together, the chromatin landscapes, DNA motif enrichment, and expression profiles of intestinal epithelial cells across developmental time suggest that HNF4 factors are more likely to function in tissue maturation than intestinal specification.

\section{HNF4 factors are dispensable for intestinal specification and villus morphogenesis, and CDX2 functions upstream of HNF4 factors}

To test the function of HNF4 in the developing gut, we deleted a conditional allele of $\mathrm{Hnf} 4 \alpha$ (Hayhurst et al., 2001) in the gut endoderm using the Shh-Cre driver (Harfe et al., 2004), which is activated in the intestinal epithelium starting at $\sim$ E9.5. We also examined germline null embryos lacking Hnf $4 \gamma$ mediated by CRISPR knockout (Chen et al., 2019), and embryos with both HNF4 paralogs simultaneously deleted (hereafter referred to $\mathrm{Hnf} 4 \alpha^{K O}, \mathrm{Hnf} 4 \gamma^{K O}$, and Hnf4 $\alpha{ }^{\text {KKO }}$, Fig.S1E,F). As we and others have reported (Banerjee et al., 2018; Gao et al., 2009; Grainger et al., 2010; Kumar et al., 2019), $C d x 2^{K O}$ in the early embryonic gut epithelium leads to an intestine exhibiting hindstomach characteristics (ATPase and foveolar PAS positive cells) in the jejunum and stratified squamous esophageal characteristics ( $\mathrm{p} 63$ positive cells) in the ileum. However, similar characteristics are not observed in the Hnf4 $\alpha \gamma^{\text {pKO }}$ embryos (Fig.2A,B), suggesting that HNF4 factors are dispensable for intestinal specification. These findings are consistent with the analysis of accessible chromatin (see above, Fig.1), in which CDX2, but not HNF4, motifs are enriched at regulatory regions most active in the embryonic intestine (Fig.1B, Fig.S1A).

Since CDX2 is required for intestinal specification but HNF4 factors appear dispensable, we hypothesized that CDX2 may function upstream of HNF4 factors. Examination of CDX2 ChIP-seq revealed that CDX2 binds to loci of $H n f 4 \alpha$ and $H n f 4 \gamma$ at E13.5 and the ChIP-seq signal strengthens at E16.5 (ChIP-seq panel in Fig.2C). Loss of CDX2 results in loss of the chromatin accessibility at the gene loci of Hnf4 factors (ATAC-seq panel in Fig.2C) and reduced Hnf4 transcript (Fig.2D) and protein levels (Fig.2E), suggesting that CDX2 is a direct activator of 
Hnf $4 \alpha$. Hnf 4 rexpression is similarly dependent upon CDX2 (Fig.2C-E); although due to the later onset of Hnf4 $\gamma$ expression, these differences first become apparent at E16.5 (Fig.2D). In developing human intestine, HNF4 factor expression is similarly dependent upon CDX2, as reflected by measurement of HNF4 transcript levels in human intestinal organoids derived from control or $C D X 2^{\text {CrisprKO }} \mathrm{hESCS}$ (Fig.2F). These results indicate that expression of HNF4 factors is dependent upon $\operatorname{CDX} 2$ during fetal maturation of the intestine.

CDX2 binds to both maturation enriched and embryo enriched regions of intestinal accessible chromatin (ATAC-seq regions from Fig.1A) at E13.5. When the intestine is mature, the CDX2 and HNF4 ChIP-seq signal is most robust at maturation enriched regions, with comparatively less binding at embryonic-accessible regions (Fig.3A,B), suggesting that these factors work to activate maturation-specific gene expression. Consistent with this idea, genes such as Myo7b and PIs1 (Fig.3C), which are bound by both HNF4 and CDX2, exhibit increased transcript expression during tissue maturation. To test whether HNF4 and CDX2 function is required for chromatin accessibility at regions that become increasingly accessible in the fetal stages, we compared ATAC-seq signal in control and mutant embryos. Chromatin accessibility is indeed compromised at maturation enriched regions from the intestinal epithelium of both E16.5 $C d \times 2^{K O}$ and $H n f 4 \alpha \gamma^{P K O}$ (Fig.4A-E). Chromatin accessibility loss in these mutants is selective to maturation-enriched chromatin, as chromatin accessiblity at promoters is unaltered, and serves as an internal control. Taken together, these data suggest an active role of CDX2 and HNF4 factors in binding and maintaining accessibility at maturation-accessible regions.

HNF4 factors thus appear dispensable for tissue specification, but their expression pattern and control of maturation-enriched regulatory regions suggest potential roles during later developmental stages. From E14.5 to E15.5, the pseudostratified intestinal epithelium resolves to a cuboidal epithelium, and mesenchymal cells crosstalk with epithelial cells to initiate villus formation (Chin et al., 2017; Walton et al., 2016a; Wells and Spence, 2014). For example, PDGFR $\alpha$-expressing mesenchymal clusters serve as signaling centers for villus morphogeneis (Walton et al., 2012). We investigated whether villus morphogenesis is disrupted upon HNF4 loss. At E15.5, Hnf4 $\alpha \gamma^{\text {PKO }}$ intestines are similar to their littermate controls (Fig.5A), and there are no obvious defects in epithelial morphology, mesenchymal condensations (PDGFR $\alpha$ positive cells), or proliferation (BrdU positive cells) upon HNF4 loss at E14.5 (Fig.5B). These results suggest that proliferation and tissue morphogenesis initiate properly in the absence of HNF4 paralogs. Consistent with the observation of normal villus morphogenesis in HNF4 mutants, adult HNF4 bound-regions (defined by ChIP-seq, Chen et al., 2019) are not very accessible during embryonic stages, but become markedly more accessible following villus morphogenesis (Fig.5C,D). Chromatin accessibility at promoter regions serves as an internal control for each timepoint (Fig.5C,D). Genes linked to HNF4-ChIP-seq regions also exhibit increased expression after villus morphogenesis, as the tissue matures (RNA-seq, Fig.5E). Together, these findings suggest that HNF4 likely functions after villus morphogenesis to drive gene expression during the maturation of the developing fetal gut.

\section{HNF4 factors are redundantly required for fetal maturation of the intestine}

From E15.5 to E18.5, villi elongate into the lumen and cells that leave the intervillus regions exit the cell cycle and begin to express markers of differentiation. To better appreciate the function 
of HNF4 factors during this timepoint, we compared ATAC-seq on E16.5 intestinal epithelial cells isolated from $\mathrm{Hnf} 4 \alpha \mathrm{p}^{\mathrm{NOO}}$ and control embryos. 5,391 accessible chromatin regions lose accessibility upon HNF4 loss (cluster 2 in Fig.6A, Table S2). Genes nearby these HNF4-dependent regions are associated with mature intestinal functions, such as adhesion, brush border formation, and lipid metabolism (Fig.6B, Table S2). For example, chromatin accessibility increases at regions of the brush border gene Enpep as the tissue matures (Fig.6C), and accessibility is almost completely lost in the $H n f 4 \alpha \gamma^{P K O}$ epithelium (Fig.6D). HNF4 paralogs directly bind to many maturation-specific genes (ChIP-seq in Fig.6E and Fig.S2), and transcript levels of these maturation-specific genes are dramatically reduced in $\mathrm{Hnf4} \alpha{ }^{\text {KKO }}$ (Fig.6F).

\section{Consistent with the loss of expression of maturation genes, $\mathrm{Hnf} 4 \alpha \gamma^{p K O}$ intestines exhibit a} translucent and distended lumen at E18.5, suggesting an underdeveloped intestine compared to their littermate controls (Fig.6G). The gross morphological defects in intestines are more severe with loss of 3 or 4 Hnf4 alleles compared to loss of 1 or 2 Hnf4 alleles (Fig.S3A). After birth, $H n f 4 \alpha \gamma^{R K O}$ pups are not able to survive, whereas $H n f 4 \alpha^{K O} \gamma^{+-}$pups can survive but exhibit growth retardation (Fig.S3B,C), which may be due to incomplete intestinal maturation. At E18.5, villi are noticeably shorter in mutants lacking 3 or $4 \mathrm{Hnf4}$ alleles but not in the Hnf4 single mutants or controls (Fig.6H, Fig.S3D), suggesting that HNF4 paralogs are redundantly required for villus elongation and extension into the developing gut lumen. There is no obvious increase in apoptosis in $\mathrm{Hnf} 4 \mathrm{\alpha} \mathrm{\gamma}^{\mathrm{PKO}}$, as evaluated by cleaved caspase 3 staining (Fig.S4A). The differentiation marker, alkaline phosphatase, which is localized to the apical surface of villus enterocytes, is normally expressed in the controls beginning at E15.5 in the maturing tissue and increases over developmental time (top panels in Fig.6I). However, alkaline phosphatase is not detectable in Hnf4 double mutants (bottom panels in Fig.6I). As expected for genetic redundancy between Hnf4 paralogs, Hnf4 single mutants show normal expression of alkaline phosphatase (Fig.S4B). Proliferative cells, which are normally restricted to the intervillus regions of the fetal gut, are expanded into the villi of $H n f 4 \alpha \gamma^{\text {KKO }}$ (Fig.S4C), which may be attributed to the loss of villus differentiation in mutants lacking HNF4 factors (Fig.6I,J). Taken together, our results indicate that HNF4 paralogs are dispensable for specification and villus morphogenesis in the developing gut, but are redundantly required for fetal maturation through direct binding to fetal maturation genes.

\section{DISCUSSION}

The regulatory mechanisms governing the transition of embryonic to mature tissue is a significant frontier for both developmental biology and regenerative medicine. Chromatin accessibility dynamics across intestinal development provide new insights into the fundamental molecular basis of intestinal specification and maturation. HNF4 motifs are most prevalent in the accessible chromatin during the maturation of the developing gut, and we provide evidence that HNF4 transcription factors are indeed important for maturation of the fetal intestine. Here, we build a model that CDX2 functions in gut specification, binds to and activates HNF4 factors in the developing gut. Together, these factors are required to mature the fetal tissue, ultimately achieving a stabilized and mature intestine. 
Interestingly, lower-level CDX2 binding in the embryo is observed at regions that will become accessible in fetal stages. (Fig.3A). Lower level CDX2 ChIP-signal at these poorly accessible regions could reflect a "low-level sampling" behavior that has recently been described for the FOXA1 transcription factor (Donaghey et al., 2018). When ectopically expressed in fibroblasts, FOXA1 exhibits low ChIP-seq signal at binding sites typically exclusive to other cell lineages, such as liver or endoderm, a behavior that could relate to FOXA1's relatively strong interaction with chromatin and slow nuclear mobility compared to other transcription factors (Sekiya et al., 2009). Low-level FOXA1 binding is strengthened at these sites by co-expression of partner factor GATA4. HNF4 factors could similarly be stabilizing CDX2 at maturation-specific regions. A potential partner for CDX2 at embryo-specific regions remains elusive. It will be interesting to test whether CDX2 exhibits similar nuclear mobility as FOXA1.

It is also interesting to note that CDX2 appears to function in both the embryonic and fetal transcription factor regulatory networks (Fig.7). Transitions between regulatory networks could more seamlessly occur when certain factors are present across the transition, such as the presence of Sox 2 and Esrrb in both embryonic stem cells and trophoblast stem cells (Adachi et al., 2013). Rather than to shut down an entire set of transcription factors and establish an entirely new set of factors, the common presence of $C D X 2$ might function as a placeholder to transition from the embryonic to fetal networks. Inhibitory factors have also been shown to play a role in tissue maturation, such as Blimp1 (Harper et al., 2011; Mould et al., 2015). collaborative networks to promote proper tissue development and function. BMP signals control intestinal villus patterning (Walton et al., 2016b) and intestinal looping (Nerurkar et al., 2017), but the functions and mechanisms of BMP signals in villus maturation are not fully explored. In a recent study, we identify a reinforcing feed-forward loop of BMP/SMAD signaling and HNF4, which promotes and stabilizes enterocyte cell identity in the adult intestine (Chen et al., 2019). Future studies can also investigate the presence of this reinforcing loop of BMP/SMAD signaling and HNF4 in the developing gut, and whether it promotes the maturation of the developing intestine or stabilization of mature transcription factor networks. These findings will build on the field's knowledge of intestinal development and may help influence efforts of regenerative medicine to provide healthy intestinal tissue to patient populations.

\section{MATERIALS AND METHODS}

\section{Mice}

The Shh-Cre transgene (Harfe et al., 2004), Cdx $2^{f / f}$ (Verzi et al., 2010), Hnf4 $\alpha^{f / f}$ (Hayhurst et al.,

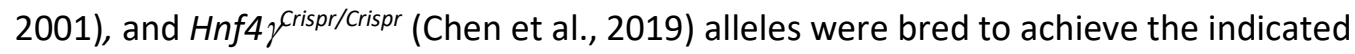
genotypes. The Shh-Cre embryos served as littermate controls unless otherwise indicated. The day on which a vaginal plug was found was considered to be E0.5. Embryonic tail biopsies were used for rapid genotyping using KAPA Mouse Genotyping Kits (Kapa Biosystems, KK7352). All mouse protocols and experiments were approved by the Rutgers Institutional Animal Care and Use Committee. All samples were collected between 12:00 and 14:00 to avoid circadian variability. 


\section{Histology and Immunostaining} Intestinal tissues were fixed with $4 \%$ paraformaldehyde at $4^{\circ} \mathrm{C}$ overnight, washed with PBS, and dehydrated through ascending alcohols prior to paraffin embedding. $5 \mu \mathrm{m}$ thick paraffin sections were used for histological staining. Hematoxylin (VWR, 95057-858) and eosin (Sigma, HT110180) staining was performed using standard procedures. Alkaline phosphatase activity was detected using the AP Staining Kit II (Stemgent). For Periodic Acid-Schiff (PAS) staining, slides were incubated in $0.5 \%$ periodic acid and stained with Schiff's Reagent (Alfa Aesar, J612171). In the case of tissue prepared for BrdU immunohistochemistry, the pregnant female mice were injected with $1 \mathrm{mg} \mathrm{BrdU}$, and embryos were harvested $1 \mathrm{~h}$ after injection. Immunohistochemistry was performed using primary antibodies against HNF4A (Santa Cruz sc6556 X, 1:2000), HNF4G (Santa Cruz sc-6558 X, 1:2000), ATPase (MBL International Corp. D0323, 1:200), P63 (Santa Cruz sc-8343, 1:500), BrdU (Bio-Rad MCA2060, 1:500), Ki67 (Abcam ab16667, 1:300), PDGFR $\alpha$ (Santa Cruz sc-338, 1:1000), Cleaved Caspase 3 (Cell Signaling 9661, 1:200), and Keratin 20 (Cell Signaling 13063, 1:2500). After incubating with secondary antibody and the Vectastain ABC HRP Kit (Vector Labs), slides were developed using 0.05\% DAB (Amresco 0430 ) and $0.015 \%$ hydrogen peroxide in $0.1 \mathrm{M}$ Tris. After mounting, the slides were viewed on a Nikon Eclipse E800 microscope. Images were photographed with a Retiga 1300 CCD (QImaging) camera using QCapture imaging software. When adjustments of sharpness, contrast, or brightness were made, they were applied uniformly for comparative images.

\section{Human embryonic stem cell (hESC) culture and differentiation}

All hESCs work was reviewed and approved by the University of Michigan human pluripotent stem cell research oversight committee (HPSCRO). The hESC cell line H9 (WA09, NIH stem registry \#0062) was obtained from the WiCell Research Institute. CDX2 ${ }^{\text {CrisprKO }}$ hESCs were generated as described previously (Kumar et al., 2019). hESCs were maintained and differentiated into endoderm, hind/midgut and human intestinal organoids as published previously (Kumar et al., 2019; Tsai et al., 2017).

\section{Intestinal epithelial cell isolation}

Embryos were collected at E18.5, and the freshly harvested embryonic small intestine (caudal stomach to rostral caecum) was opened longitudinally with forceps, cut into $1 \mathrm{~cm}$ pieces, and then rotated in $3 \mathrm{mM}$ EDTA in PBS at $4{ }^{\circ} \mathrm{C}$ for $40 \mathrm{~min}$. To release the epithelial cells from underlying muscular tissue, the tissue was vigorously shaken after EDTA incubation. The supernatant was collected as the whole epithelium fraction. Cells were pelleted by centrifugation at $170 \mathrm{~g}$ at $4{ }^{\circ} \mathrm{C}$ and then washed with cold PBS, and processed for RNA extraction using Trizol (Invitrogen, 15596018) according to the manufacturer's protocols.

\section{RNA extraction and qRT-PCR}

The RNA was reverse transcribed using SuperScript III First-Strand Synthesis SuperMix (Invitrogen, 18080-400) with Oligo(dT) 20 primers to prepare cDNA. qRT-PCR analysis was performed using gene-specific primers and SYBR Green PCR Master Mix (Applied Biosystems, 4309155). The sequences of the primers used are available upon request. The $2^{-\Delta \Delta C t}$ method was applied to calculate the fold change of relative transcript levels, and Hprt1 was used for normalization.

\section{Single intestinal epithelial cell isolation for ATAC-seq}

Embryos were collected at E16.5, and the freshly harvested embryonic small intestine (caudal stomach to rostral caecum) was opened longitudinally with forceps and cut into $1 \mathrm{~cm}$ pieces. Intestinal tissues were treated with pre-warmed $0.25 \%$ Trypsin for $8 \mathrm{~min}$ at $37^{\circ} \mathrm{C}$ on a vortex 
station (speed set between 6-7), neutralized with $10 \%$ FBS, and passed through a $70-\mu \mathrm{m}$ cell strainer. Cells were stained with the anti-CD326 (EPCAM) magnetic microbeads antibody (Miltenyi Biotec, 130-105-958) for 40 min on ice. To obtain single magnetic antibody conjugated EpCAM positive epithelial cells, stained cells were passed through a 40- $\mu \mathrm{m}$ cell strainer and then collected over a MS column (Miltenyi Biotec, 130-042-201) in a magnetic field. 20,000 cells were used for ATAC-seq as described previously (Buenrostro et al., 2013; Buenrostro et al., 2015) with slight modifications. Briefly, cells were resuspended in ice-cold lysis buffer ( $10 \mathrm{mM}$ Tris, $\mathrm{pH} 7.4$, $10 \mathrm{mM} \mathrm{NaCl}, 3 \mathrm{mM} \mathrm{MgCl}$, and $0.1 \% \mathrm{NP}-40$ ), and then centrifuged at $500 \mathrm{~g}$ for $10 \mathrm{~min}$ at $4^{\circ} \mathrm{C}$. The isolated nuclei were incubated with Nextera Tn5 Transposase (Illumina FC-121-1030) at $37^{\circ} \mathrm{C}$ for $30 \mathrm{~min}$. The transposed chromatin was purified with QIAquick PCR Purification Kit (QIAGEN), and PCR was amplified with high-fidelity 2X PCR Master Mix (New England Biolabs M0541). One-third of the maximum fluorescent intensity was used to determine the additional cycles. The PCR amplified libraries were purified, and fragment size was selected using Pippin Prep and sequenced on Illumina NextSeq 550.

\section{Bioinformatics analysis} For ATAC-seq and ChIP-seq analysis, raw sequencing reads (fastq) were quality checked using fastQC (v0.11.3) and were further aligned to mouse ( $\mathrm{mm9}$ ) genomes using bowtie2 (v2.2.6) (Langmead and Salzberg, 2012) to obtain bam files. Deeptools bamCoverage (Ramirez et al., 2016) (v2.4.2, duplicate reads ignored, RPKM normalized and extended reads) was used to generate bigwig files from bam files, and BigWigMerge (v2) was used to merge the bigwig files of different replicates. MACS (v1.4.1) (Zhang et al., 2008) was used for peak calling and to generate bed files from bam files, and BEDTools (v2.17.0) (Quinlan, 2014) was used to merge, intersect or subtract the intervals of bed files. Promoters were defined within $2 \mathrm{~kb}$ of the transcription start sites of RefSeq genes, and enhancers were defined by excluding promoters. Haystack (v0.4.0) (Pinello et al., 2018) quantile normalized bigwigs were used to create $k$-means clustering heatmaps of ATAC-seq using computeMatrix and plotHeatmap from deeptools (v2.4.2) (Ramirez et al., 2016). Genomic regions of desired $k$-means clusters were extracted from bed files generated by plotHeatmap for further analysis. Homer findMotifsGenome.pl (v4.8.3, homer de novo Results) (Heinz et al., 2010) was used to identify transcription factor motifs enriched at peaks. Genes associated with peaks were identified using the peak2gene/BETAminus function (v1.0.2) in Cistrome tools (Liu et al., 2011). Enriched gene ontologies were identified from genomic regions (bed file) using GREAT analysis (v3.0.0) (McLean et al., 2010) or DAVID (v6.8) (Huang da et al., 2009). SitePro (v1.0.2) (Shin et al., 2009) was used to visualize the average signals of ChIP-seq in the desired genomic regions. The Integrative Genomics Viewer (IGV 2.4.13) (Robinson et al., 2011) was used to visualize bigwig tracks.

For RNA-seq analysis, raw sequencing reads (fastq) were quality checked using fastQC (v0.11.3), and were further aligned to mouse (mm9) genomes using Tophat2 (v2.1.0) (Kim et al., 2013) to generate bam files. Kallisto (v0.44.0) (Bray et al., 2016) was utilized to quantify the transcript abundances of the RNA-Seq samples through pseudoalignment, using single-end reads and an Ensembl mm9 transcriptome build index. Then, the tximport (v1.8.0) (Soneson et al., 2015) package was run in $\mathrm{R}$ (v3.5.2) to create gene-level count matrices for use with DESeq2 (v1.20) (Love et al., 2014) by importing quantification data obtained from Kallisto. DESeq2 was then used to generate RPKM values per kilobase of gene length per million mapped fragments at each time course point with comparison of $C d x 2^{K O}$ replicates and wild-type replicates. DESeq2 was also used to generate $p$-values of gene matches. Genes with FPKM $>1$, a commonly used 
was used to display relative transcript levels of genes of interest by using normalized RPKM values. The Integrative Genomics Viewer (IGV 2.4.13) (Robinson et al., 2011) was used to visualize bam tracks.

\section{Statistical analysis} The data is presented as mean \pm SEM, and statistical comparisons were performed using twosided Student's t-test at $P<0.001^{* * *}, P<0.01^{* *}$ or $P<0.05^{*}$. Bioinformatics related statistical analysis was done with the embedded statistics in each package, including HOMER (Heinz et al., 2010), GREAT (McLean et al., 2010), DAVID (Huang da et al., 2009) and DESeq2 (Love et al., 2014). $P<0.05$ (95\% confidence interval) was considered statistically significant.

\section{Acknowledgements}

We thank Madhurima Saxena and Ramesh Shivdasani for helpful suggestions and curating published data. We thank Kenneth Zaret for helpful discussions.

\section{Competing interests}

The authors declare no competing or financial interests.

\section{Author contributions}

L.C. conceived and designed the study, performed benchwork and bioinformatics, collected and analyzed the data, and wrote the manuscript; N.H.T., S.L., R.P.V. and A.P. contributed to benchwork. R.A. contributed to bioinformatics; J.R.S and Y-H.T. provided human intestinal organoid data; M.P.V. conceived, designed and supervised the study, and wrote the manuscript.

\section{Funding}

This research was funded by a grant from the NIH (R01CA190558, M.P.V.). J.R.S and M.P.V are also supported by the Intestinal Stem Cell Consortium from the National Institute of Diabetes and Digestive and Kidney Diseases (NIDDK) and National Institute of Allergy and Infectious Diseases (NIAID) of the National Institutes of Health under grant number U01 DK103141. Support was also received from the Sequencing Facility of the Rutgers Cancer Institute of New Jersey (P30CA072720) and the University of Michigan Center for Gastrointestinal Research (UMCGR) (NIDDK 5P30DK034933). L.C. was supported by New Jersey Commission on Cancer Research grant (DFHS18PPC051). N.H.T., S.L., R.P.V. and A.P. were supported by MacMillan Summer Undergraduate Research Fellowships.

\section{Data availability}

All ATAC-seq data of this study have been deposited in GEO (GSE128674). The following secure token has been created to allow review of record GSE128674: cpgbwsyarrghpeh. The following datasets from GEO were reanalyzed: The accession numbers for the transcriptome and chromatin accessibility of time-course WT and $C d \times 2^{K O}$ from our previous studies are GSE115314 (Kumar et al., 2019) and GSE115541 (Banerjee et al., 2018). The accession numbers for the CDX2 ChIP-seq and HNF4 ChIP-seq from our previous studies are GSE34568 (Verzi et al., 2013), GSE115314 (Kumar et al., 2019) and GSE112946 (Chen et al., 2019). GSE89684 (Kazakevych et al., 2017) was used to mark active chromatin with the time-course H3K27ac ChIP-seq. The accession number for the RNA-seq data of differentiation of hESCs into human intestinal 


\section{Supplementary information}

425 Table S1. Genome coordinates for ATAC-seq performed in intestinal epithelial cells from E11.5

426 embryo to adult, including 30,702 embryonic enhancer regions (cluster 3 from Fig.1A) and

427 10,544 maturation enhancer regions (cluster 2 from Fig.1A). Additionally, the full list of HOMER

428 de novo motif-calling analysis on these embryonic and maturation enriched regions are reported

429 respectively. Finally, the results of GO term enrichment using GREAT analysis for genes with

430 their transcriptional start sites within $20 \mathrm{~kb}$ of these embryonic and maturation regions are

431 reported respectively. These data correspond to findings in Fig.1.

Table S2. ATAC-seq performed in intestinal epithelial cells from E16.5 Hnf4 $\alpha \gamma^{p K O}$ versus $\mathrm{Hnf4} \gamma^{+/-}$ controls. Genome coordinates for 5,391 accessible chromatin regions become inaccessible upon HNF4 loss (cluster 2 from Fig.6A). Additionally, the full list of HOMER de novo motif-calling analysis on these HNF4 chromatin-dependent regions are reported. Finally, the results of GO term enrichment using DAVID analysis for genes with their transcriptional start sites within 20 $\mathrm{kb}$ of these HNF4 chromatin-dependent regions are reported. These data correspond to findings in Fig.6. 
bioRxiv preprint doi: https://doi.org/10.1101/610428; this version posted April 15, 2019. The copyright holder for this preprint (which was not certified by peer review) is the author/funder, who has granted bioRxiv a license to display the preprint in perpetuity. It is made available under aCC-BY-NC-ND 4.0 International license.

A

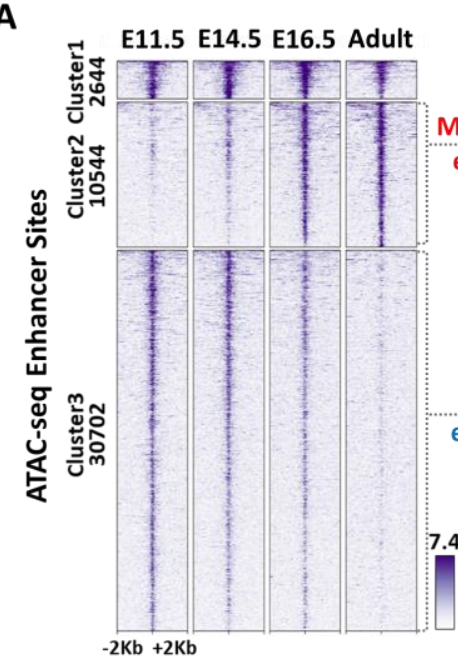

D

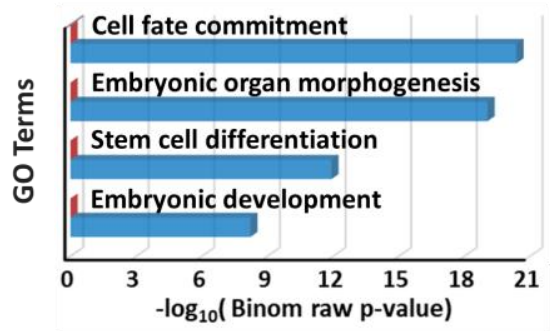

B

Cluster2

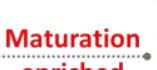

enriched

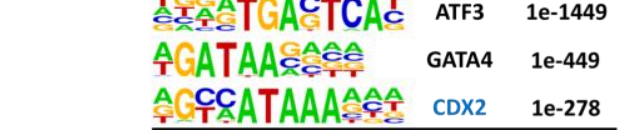

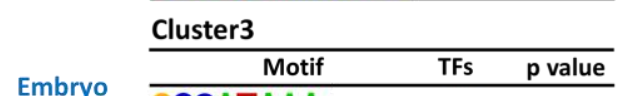

enriched

enriched

.4

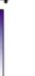

Fig. 1

C
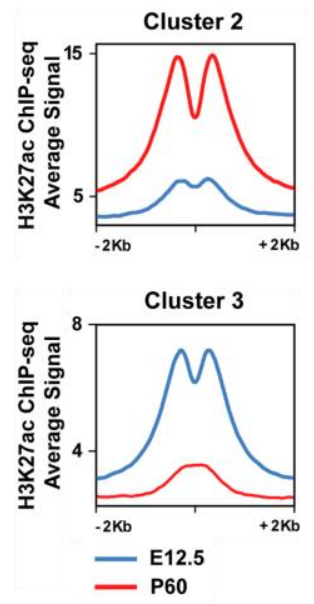

E
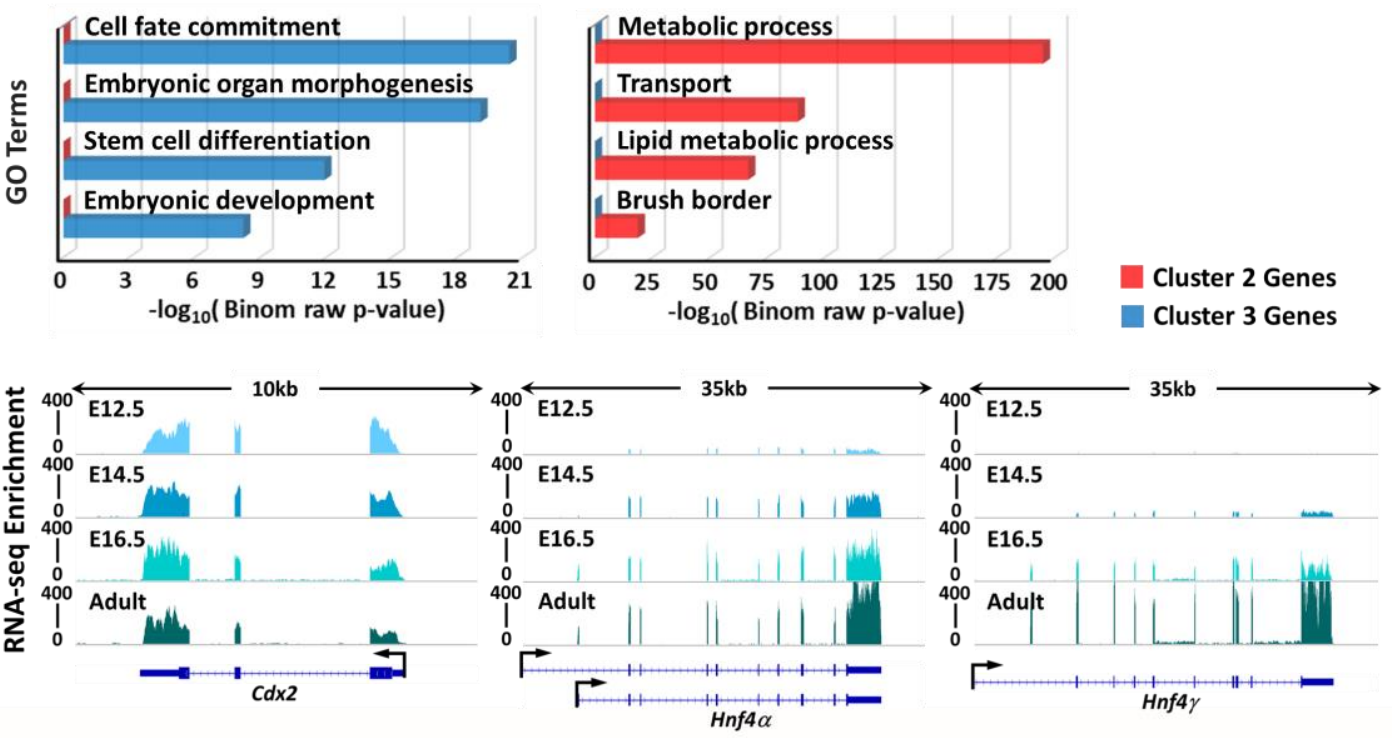

F
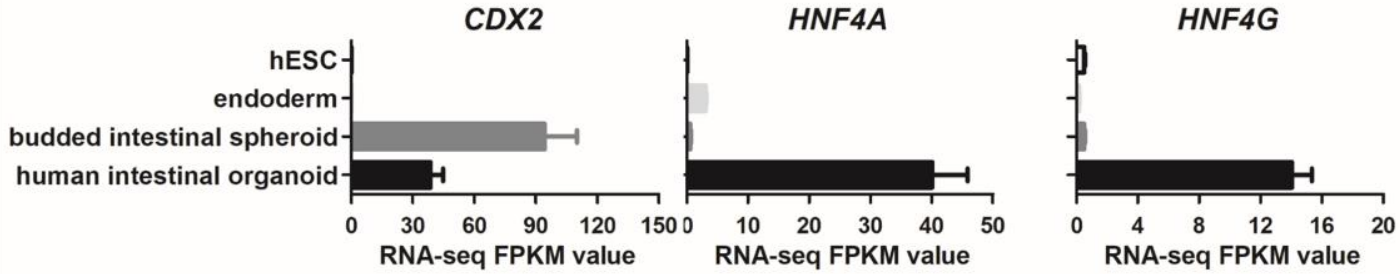

Fig. 1. Chromatin landscapes indicate that HNF4 factors function in maturation of the developing gut. (A) ATAC-seq (GSE115541, $n=2$ biological replicates per timepoint, isolated embryonic epithelium was collected from the entire small intestine) defined regions of accessible chromatin across indicated stages of mouse intestinal epithelium development (MACS $P$ value $\leq 10^{-5}$ ). $K$-means clustering analysis reveals a shift in accessible enhancer chromatin of intestinal epithelial cells across developmental time. (B) HOMER de novo DNA- 
452 sequences are more prevalent in accessible regions of the early embryonic state (embryo

453 enriched, cluster 3), whereas HNF4 binding sequences are more prevalent in accessible regions

454 of the fetal and adult states (maturation enriched, cluster 2). (C) H3K27ac ChIP-seq (GSE89684, $n$

$455=2$ biological replicates per timepoint) profiles demonstrate that the active chromatin marker is

456 enriched in a stage-specific manner, corresponding to embryo-specific or P60 adult-specific

457 accessible regions. (D) GREAT GO term analysis shows distinct gene ontologies of target genes

458 linked to stage-specific ATAC-seq sites within 20kb. (E) RNA-seq of purified epithelium

459 (GSE115541, $\mathrm{n}=2$ biological replicates per timepoint, isolated embryonic epithelium was

460 collected from the entire small intestine) shows that $C d \times 2$ is highly and equally expressed across

461 developmental time, whereas $H n f 4 \alpha$ and $H n f 4 \gamma$ are not robustly expressed until E14.5 and

462 E16.5, respectively. (F) RNA-seq (E-MTAB-4168) shows that CDX2 expression is induced when

463 human endoderm is specified to intestine by treatment with FGF4 $(500 \mathrm{ng} / \mathrm{ml})$ and CHIR99021

464 (2 $\mu \mathrm{M}$, WNT agonist). By contrast, HNF4A/G expression is not induced until human intestinal

465 organoids are formed by subsequent differentiation steps. See schematic in Fig.S1D for details

466 on human intestinal organoid differentiation conditions. Data are presented as mean $\pm \mathrm{SEM}$. At

467 least 3 biological replicates are included per stage, and samples from the same stage are

468 grouped and presented.

469

470 
Fig. 2

A
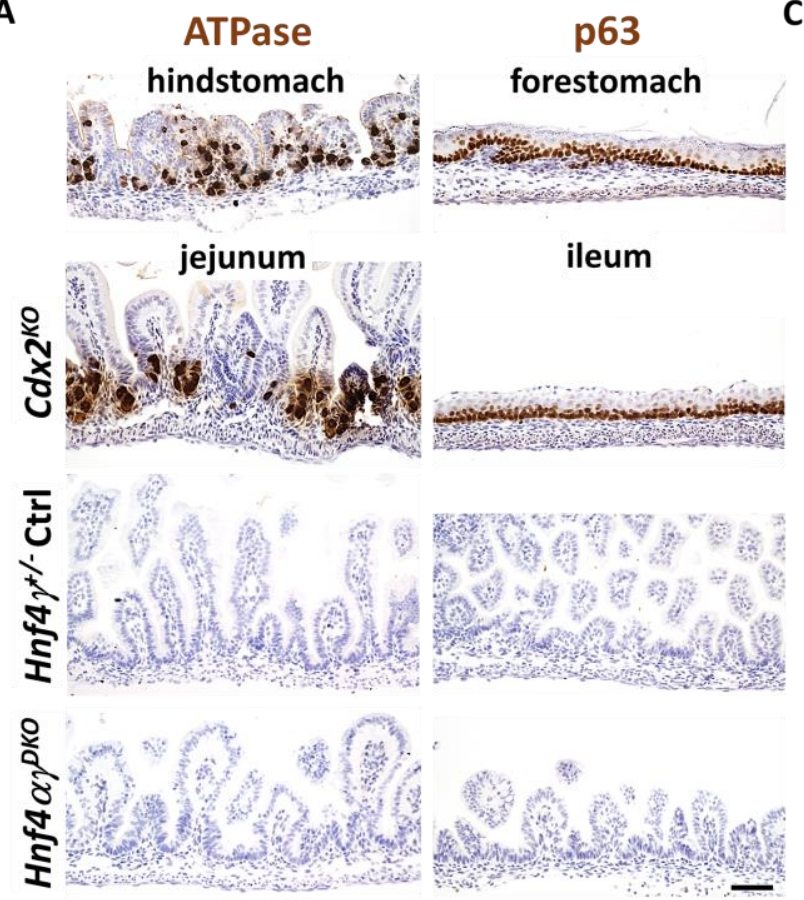

C

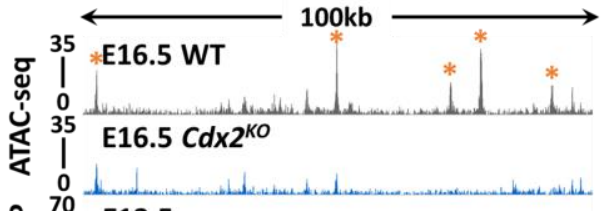

온

E13.5

Uิ

${ }_{0}^{70}$

0
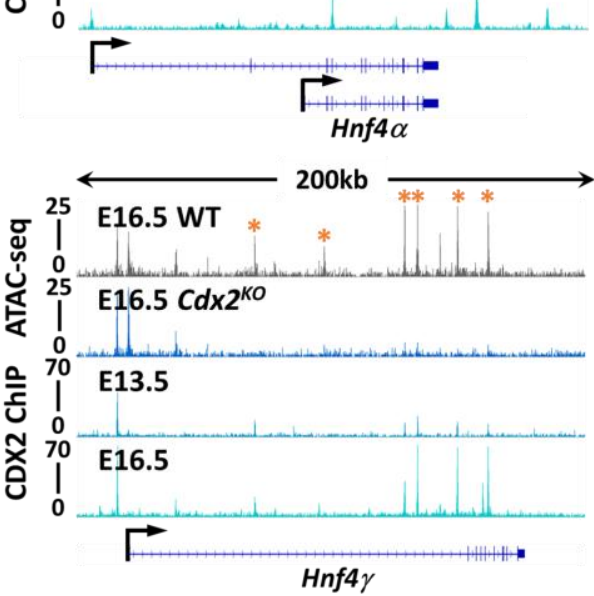

B
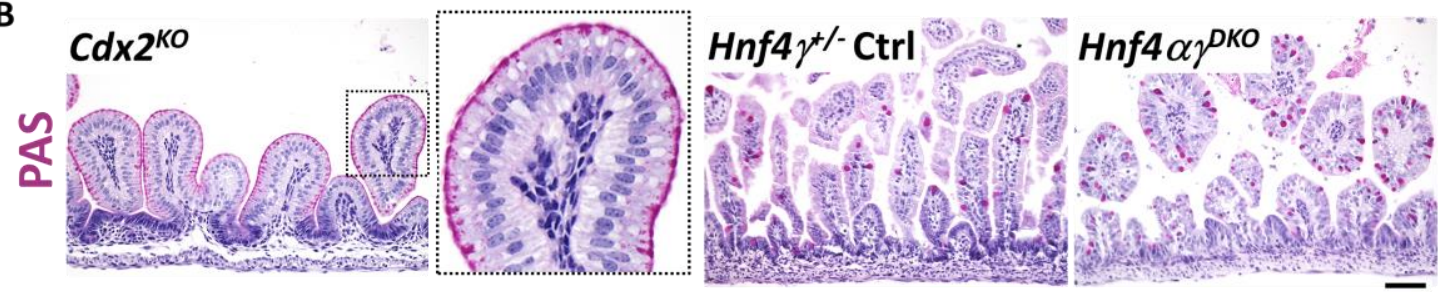

D

E
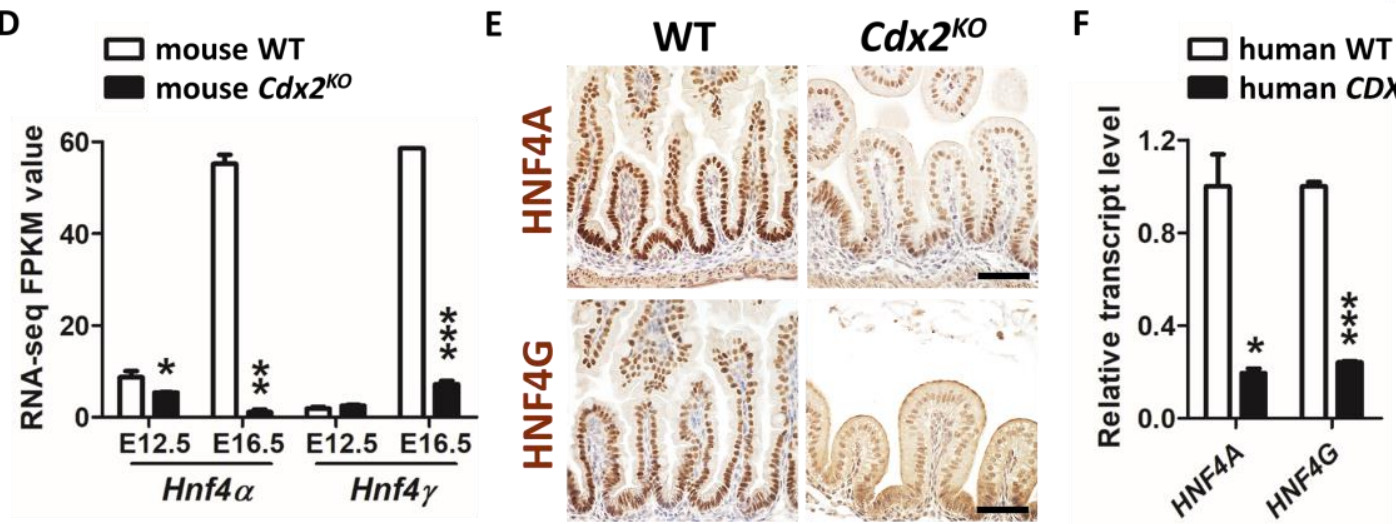

Fig. 2. HNF4 is activated by CDX2 in the developing gut, but HNF4 is not required for intestinal specification. (A) Immunostaining of ATPase and p63 (representative of 4 biological replicates)

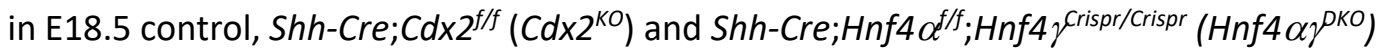
embryos. The hindstomach marker ATPase is ectopically expressed in the $C d \times 2^{\mathrm{KO}}$ jejunum but not in the control and $H n f 4 \alpha \gamma^{p K O}$ jejunum. The forestomach marker p63 is ectopically expressed in the $C d \times 2^{K O}$ ileum (squamous mucosa) but not in the control and $H n f 4 \alpha \gamma^{K O}$ ileum. (B) PAS staining indicates that normal intestinal goblet cells are replaced with cells resembling gastric foveolar cells (PAS positive cells at apical cell surface) in the $C d \times 2^{K O}$ jejunum of E18.5 embryos, 
480 but not in the control or Hnf4 $\alpha{ }^{p K O}$ jejunum (representative of 4 biological replicates). (C) CDX2

481 binds to Hnf4 $\alpha$ and Hnf4 $\gamma$ loci at E13.5 and E16.5 (CDX2 ChIP, GSE115314, $\mathrm{n}=2$ biological

482 replicates, whole small intestine epithelium), and accessible chromatin is compromised at gene

483 loci of $H n f 4 \alpha$ and Hnf4 $\gamma$ upon CDX2 loss (ATAC-seq, GSE115314, $\mathrm{n}=2$ biological replicates,

484 whole small intestine epithelium). Asterisks denote putative regulatory regions. (D) The

485 transcript levels of $H n f 4 \alpha$ and $H n f 4 \gamma$ are significantly downregulated in the intestinal epithelial

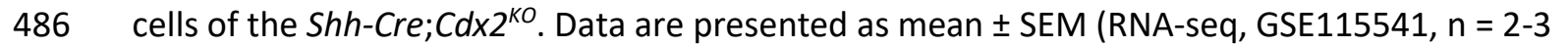

487 biological replicates, whole small intesitne epithelium). Statistical tests are embedded in DESeq2

488 at $P<0.001^{* * *}, P<0.01^{* *}$ and $\left.P<0.05^{*}\right)$. (E) Immunostaining of HNF4A and HNF4G shows

489 reduced protein levels of HNF4 paralogs in E18.5 $C d x 2^{K O}$ (representative of 4 biological

490 replicates). Scale bars, $50 \mu \mathrm{m}$. (F) qRT-PCR shows reduced transcript levels of HNF4A and HNF4G

491 in human intestinal organoids derived from $C D X 2^{\text {CrisprKO }} \mathrm{hES}$ cells compared to organoids derived

492 from control cells. Data are presented as mean \pm SEM $(n=3$ biological replicates, Student's t-

493 test, two-sided at $\mathrm{P}<0.001^{* * *}$ and $P<0.05^{*}$ ).

494

495 
A

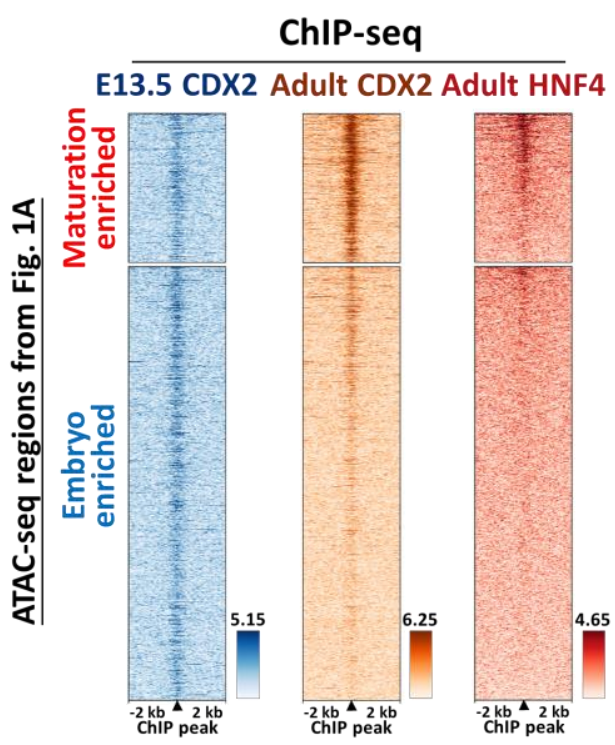

B

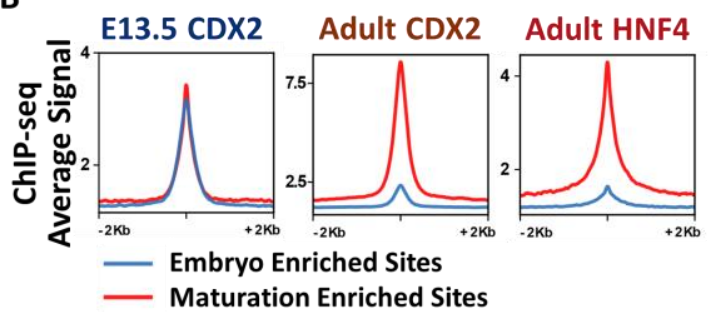

C

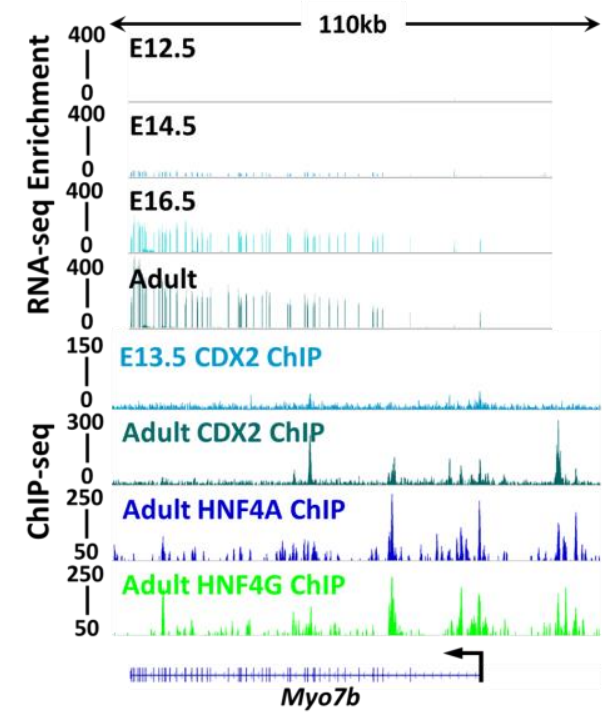

Fig. 3

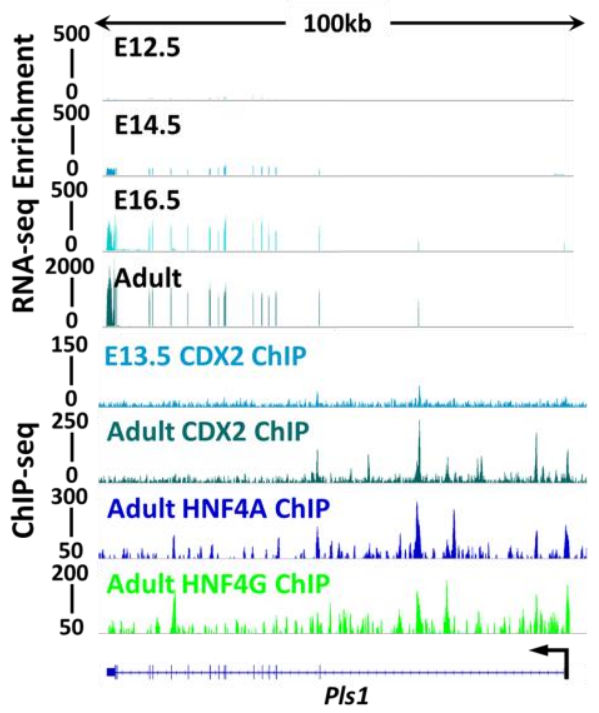

Fig. 3. Chromatin regions that become accessible in the fetal tissue are bound by CDX2 and HNF4. Genes nearby these regions are activated at fetal stages. (A-B) ChIP-seq profiles show that CDX2 (GSE115314, $n=2$ biological replicates, whole small intestine epithelium) binds to both maturation enriched (cluster 2 of Fig.1A) and embryo enriched (cluster 3 of Fig.1A) regions of intestinal accessible enhancer chromatin in E13.5 embryos. When tissues become mature, CDX2 (GSE34568 and GSE115314, $\mathrm{n}=2$ biological replicates) and HNF4 (GSE112946, $\mathrm{n}=2$ biological replicates per HNF4A and HNF4G ChIP, adult duodenum epithelium) bind more robustly to the maturation enriched regions rather than to the embryo enriched regions. The peaks of CDX2 ChIP in the heatmaps are aligned to the binding events of HNF4 ChIP. (C) Examples of genes located at maturation enriched regions are visualized using IGV. Brush border genes, such as Myo7b and Pls1, are expressed when maturation occurs at E16.5 and are robustly expressed when intestines become mature in the adult (RNA-seq panels, GSE115541, $n=2$ biological replicates). These genes are directly bound by CDX2 (GSE34568 and GSE115314, n = 2 biological replicates) and HNF4 (GSE112946, $n=2$ biological replicates per HNF4A and HNF4G ChIP). 
Fig. 4

A

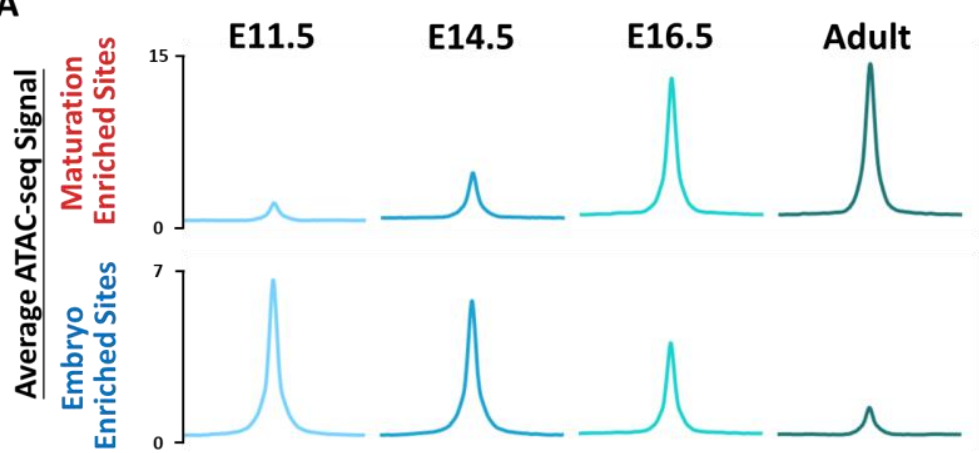

B

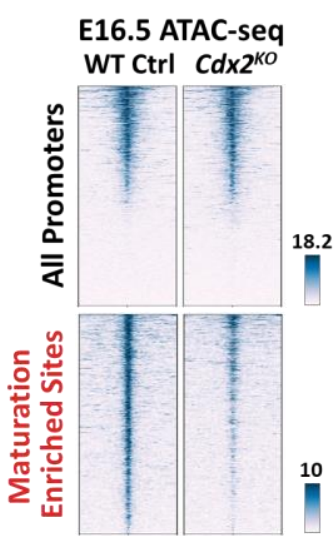

C

D
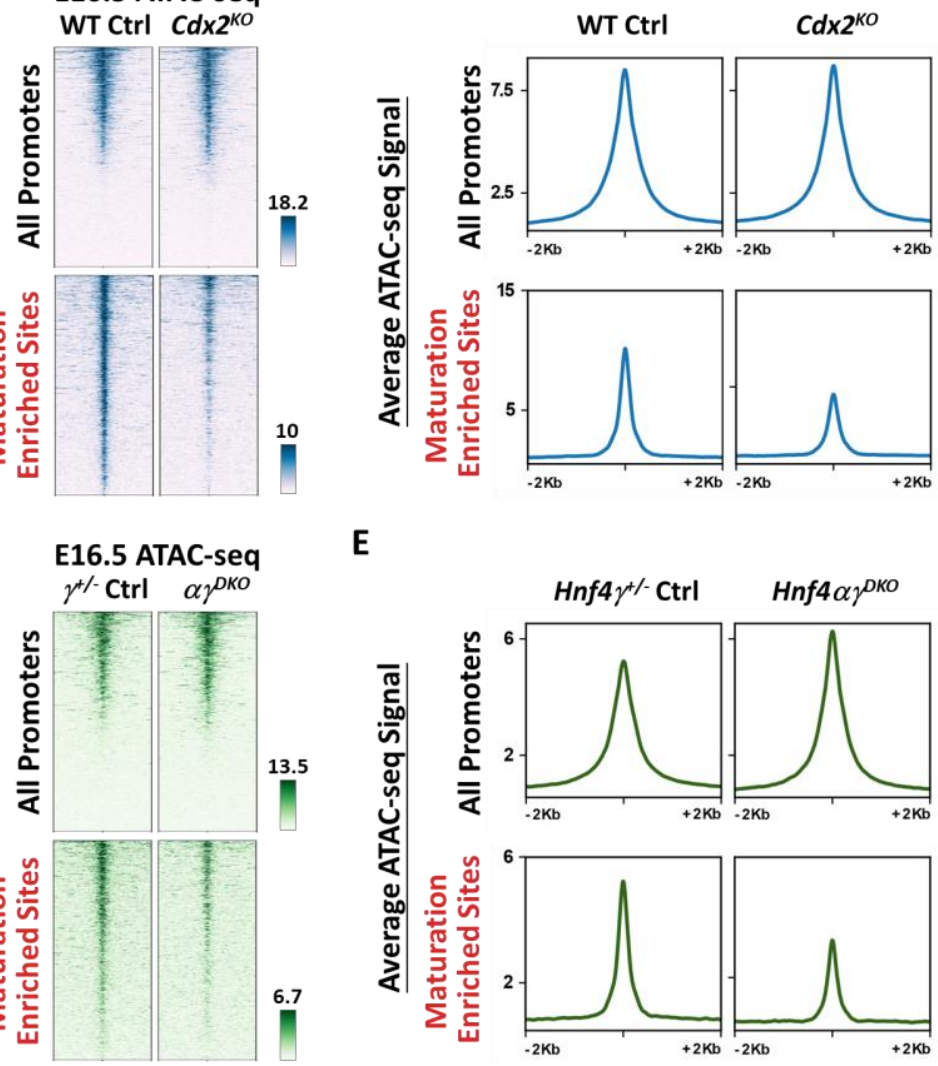

E

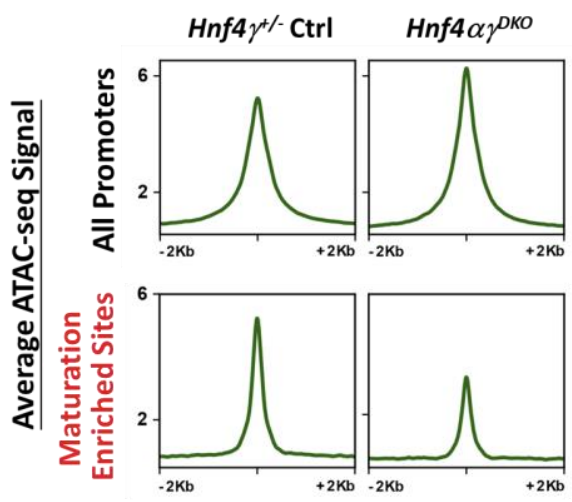

Fig. 4. Maturation-enriched regions of accessible enhancer chromatin show loss of accessibility in the intestinal epithelium of $C d \times 2^{K O}$ and $H n f 4 \alpha \gamma^{p K O}$ mutants. (A) The intestinal chromatin (GSE115541, $\mathrm{n}=2$ biological replicates) becomes more accessible at maturation enriched regions and less accessible at embryo enriched regions (ATAC-seq regions defined from Fig.1A) from E11.5 embryo to adult. The intestinal chromatin accessibility at the maturation enriched sites is compromised upon depletion (Shh-Cre) of Cdx2 (B-C, GSE115314) or Hnf4 (D-E) in E16.5 embryos, whereas promoter regions are relatively unaffected and serve as an internal control ( $\mathrm{n}=2 \mathrm{WT}$ controls, $1 \mathrm{Hnf4} \gamma^{+/-}$controls, $2 \mathrm{Cd} \times 2^{K O}$ mutants and $2 \mathrm{Hnf} 4 \alpha \gamma^{\text {TKO }}$ mutants, whole small intestine epithelium). 
A

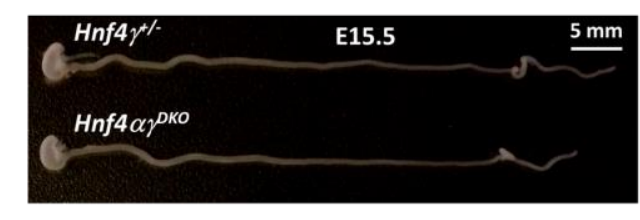

B
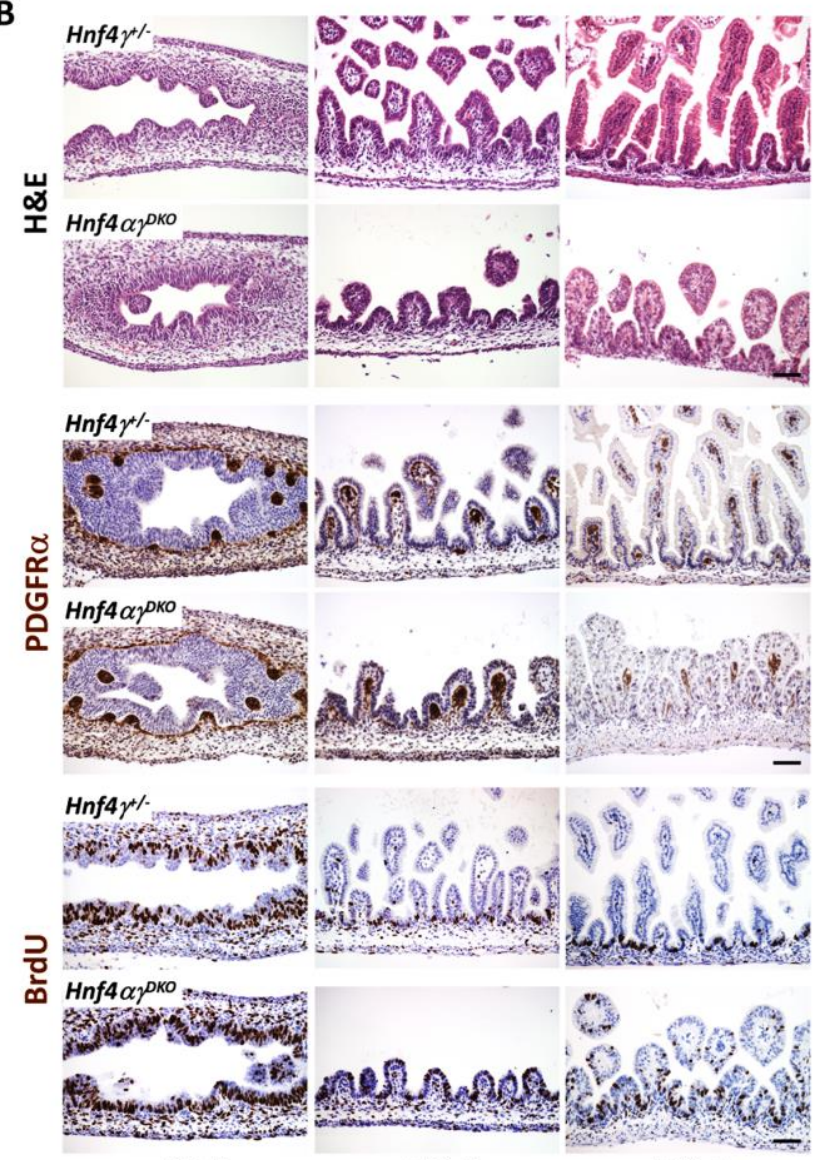

E14.5
E16.5
E18.5
D

Fig. 5

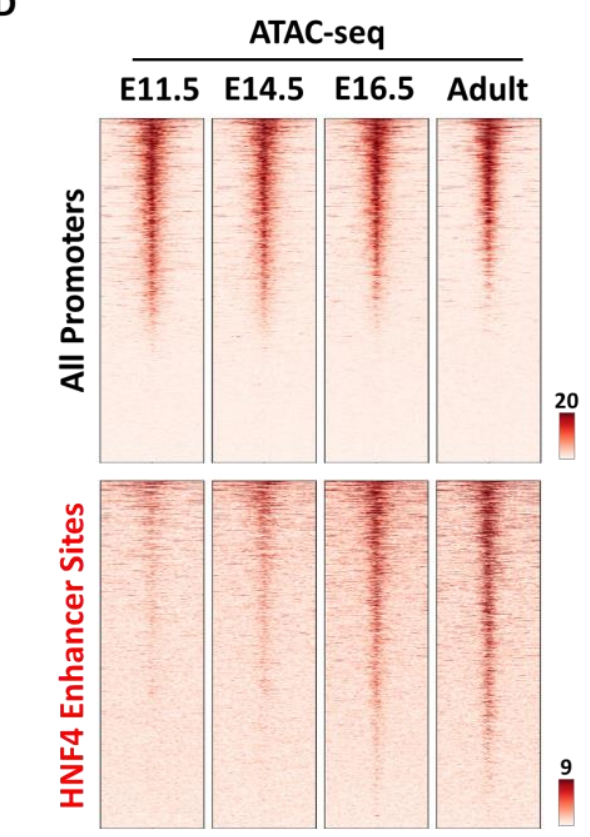

E

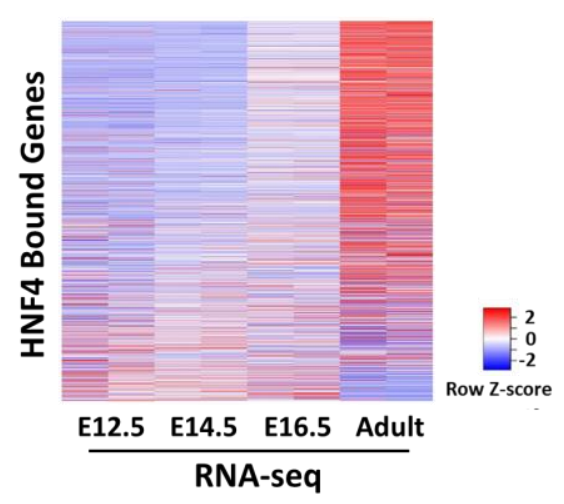

C

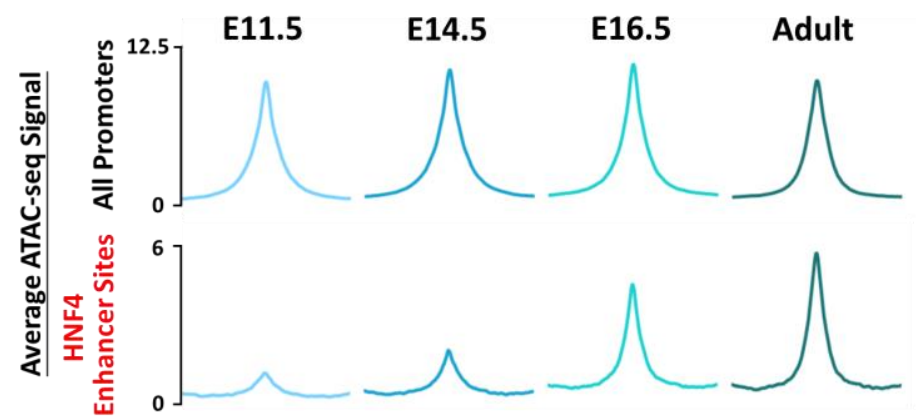

Fig. 5. Villus morphogenesis initiates despite the loss of HNF4, and chromatin accessibility and gene regulation at HNF4-binding sites suggest a role for HNF4 after villus morphogenesis. (A) Whole mount images of representative intestines dissected from $\mathrm{Hnf} 4 \alpha \mathrm{P}^{\mathrm{DKO}}$ and littermate $H n f 4 \gamma^{+-}$control embryos at E15.5 ( $n=4$ biological replicates per genotype). (B) H\&E staining and immunostaining of PDGFR $\alpha$ and BrdU across developmental time show no striking differences, 
531 indicating that HNF4 factors are dispensable for the onset of villus morphogenesis

532 (representative of 4 biological replicates). The pregnant female mice were injected with $1 \mathrm{mg}$

533 BrdU $1 \mathrm{~h}$ before euthanasia. Scale bars, $50 \mu \mathrm{m}$. (C-D) Chromatin becomes accessible during fetal

534 and adult stages at enhancer regions bound by HNF4 in the adult epithelium (MACS $P$ value $\leq 10^{-}$

$535{ }^{3}$, GSE112946, $\mathrm{n}=2$ biological replicates per ChIP), indicating a potential role for HNF4 in

536 activating genes nearby these regions during intestinal maturation. (E) Increased transcript

537 levels of HNF4-bound genes in intestinal epithelial cells are observed across developmental time

538 from E12.5 to adult villi. Genes with transcriptional start sites within 20 kb of HNF4 enhancer

539 bound sites were used for plotting a heatmap showing the relative transcript expression levels

540 over the indicated developmental stages (GSE115541, $n=2$ biological replicates per timepoint). 


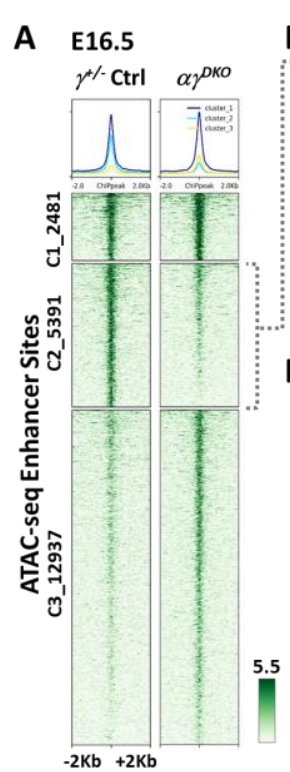

G
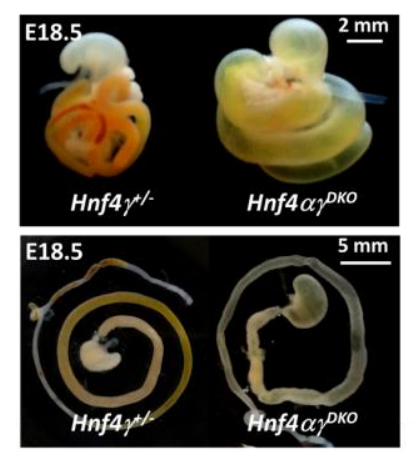

,

B Genes linked to HNF4 Chromatin-dependent Regions

$\mathbf{F}$

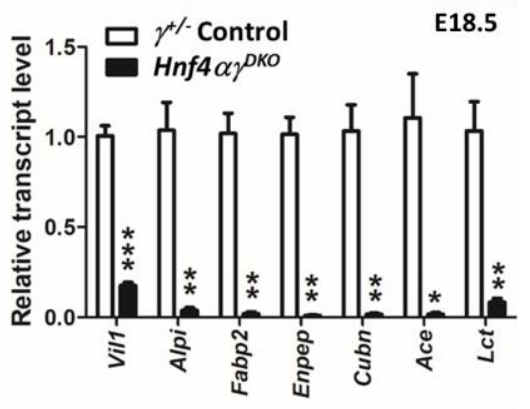

H

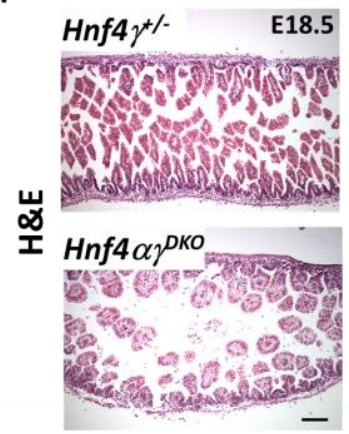

C

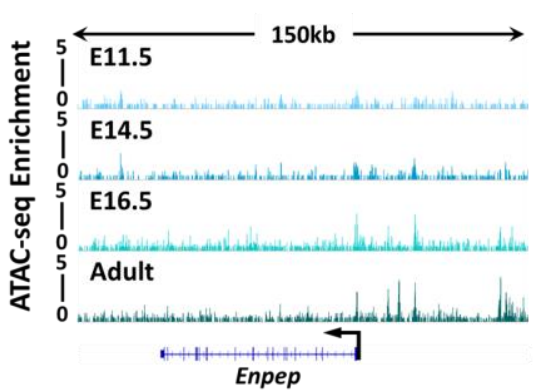

D

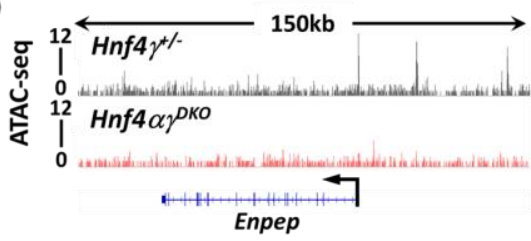

\section{$\mathrm{E}$}

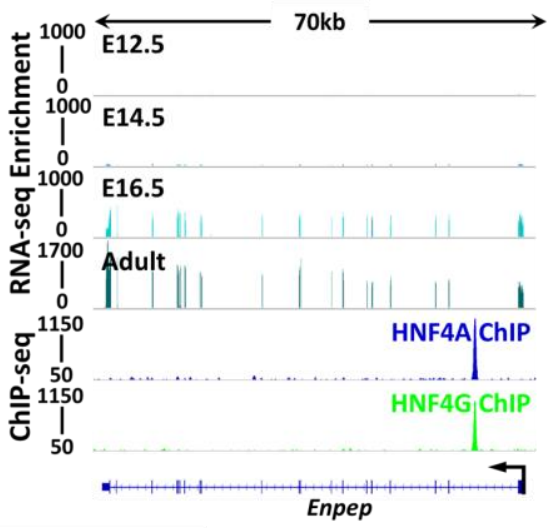

J $\quad H n f 4 \gamma^{+\%} E 18.5$

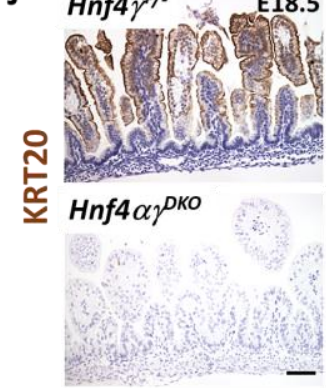

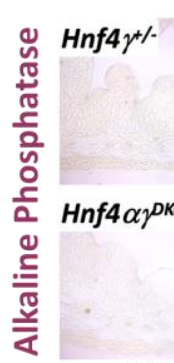

E14.5

E15.5

E16.5

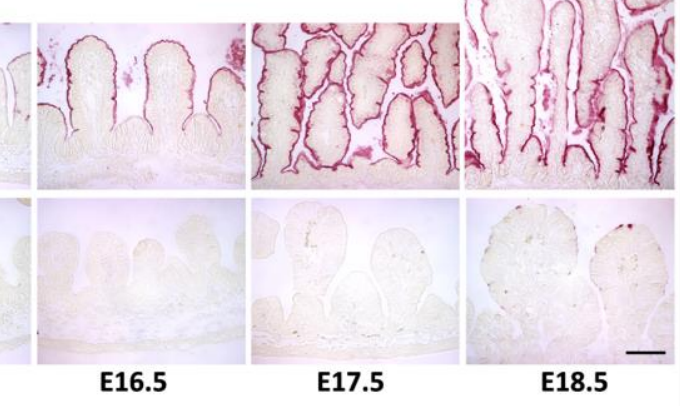

E18.5

546 Fig. 6. HNF4 paralogs are redundantly required for fetal maturation of the intestine. (A) $K$ -

547 means clustering of ATAC-seq data collected from E16.5 intestinal epithelial cells isolated from

$548 H n f 4 \alpha \gamma^{\text {KKO }}$ and control embryos identify 5,391 regions (cluster 2) that are dependent on HNF4

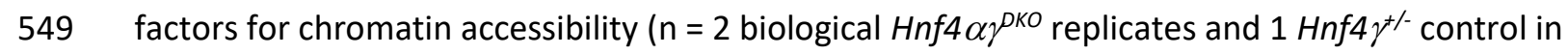

550 this study; MACS $P$ value $\leq 10^{-5}$ ). (B) Functional annotation of the genes linked to HNF4-

551 dependent accessible chromatin by DAVID. Genes with transcriptional start sites within $20 \mathrm{~kb}$ of

552 ATAC-seq sites of cluster 2 (loss of chromatin accessibility upon HNF4 loss) from panel 6A were

553 used for analysis. (C-E) Examples of chromatin accessibility at HNF4 dependent ATAC-seq sites

554 are visualized using IGV. (C) ATAC-seq (GSE115541) shows a time-dependent increase of 
chromatin accessibility at locus of the brush border gene Enpep from E11.5 embryo to adult. (D) ATAC-seq shows compromised chromatin accessibility at the Enpep locus is observed in E16.5 Hnf4 $\alpha{ }^{\text {PKO }}$. (E) RNA-seq (GSE115541) shows corresponding increase in transcript levels of Enpep over developmental time, and HNF4A and HNF4G directly bind to Enpep (GSE112946, ChIP-seq panel), suggesting a direct regulation. (F) qPCR shows that the transcript levels of genes known to be expressed in the mature intestine are dramatically reduced in $H n f 4 \alpha{ }^{\text {KKO }}$ compared to that of the littermate $\mathrm{Hnf} \gamma^{+/-}$controls in isolated E18.5 intestinal epithelial cells. Data are presented as mean \pm SEM ( $n=4$ controls and 5 mutants, Student's t-test, two-sided at $P<0.001^{* * *}, P<$ $0.01^{* *}$ and $P<0.05^{*}$ ). (G) Whole mount images of E18.5 intestine indicate that loss of HNF4 paralogs leads to an underdeveloped intestine with distended and translucent lumen (representative of 6 biological replicates). (H) Strikingly stunted villi are observed in E18.5 $\mathrm{Hnf} 4 \alpha{ }^{p K O}$ embryos compared to littermate $\mathrm{Hnf} 4 \gamma^{+/-}$controls, as revealed by H\&E staining (representative of 4 biological replicates, E18.5 duodenum; see expanded panel in Fig.S3D). (I) Hnf $4 \alpha{ }^{p K O}$ exhibits diminished alkaline phosphatase staining (differentiation marker) across developmental time (representative of 4 biological replicates, E14.5-E18.5 duodenum). (J) Keratin 20 immunostaining also indicates compromised intestinal differentiation in $\mathrm{Hnf4} \alpha \mathrm{\gamma}^{\text {pKO }}$

Fig. 7. Potential model of how intestinal transcription factor networks shift as embryos mature to adults. Left, CDX2 functions in gut specification, and robustly binds to embryo enriched accessible chromatin regions with other embryonic transcription factors. In the

\section{Embryo enriched chromatin}

\section{Embryo}

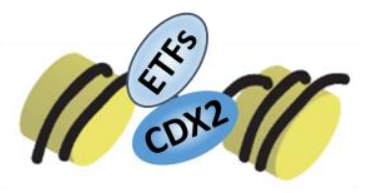

Maturation enriched chromatin

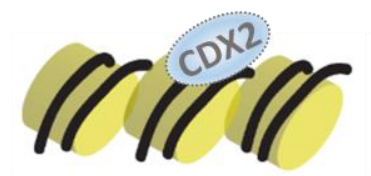
embryo, lower-level CDX2 binding may also occur at regions that will become accessible in the fetus. Right, as intestine matures to fetal stages, maturation-enriched regions become more
Fig. 7

\section{Maturation}
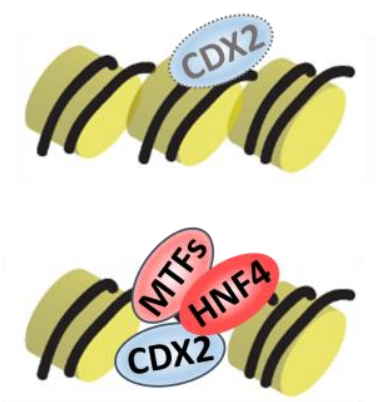
ETFs: Embryonic transcription factors
MTFs: Maturation transcription factors accessible. A new transcription factor network, highlighted by HNF4 works together to stabilize enhancer chromatin and drive intestinal maturation. 
bioRxiv preprint doi: https://doi org/10.1101/610428. this version posted April 15,2019 . The copyright holder for this preprint (which was not certified by peer review) is the author/funder, who has granted bioRxiv a license to display the preprint in perpetuity. It is made available under aCC-BY-NC-ND 4.0 International license.

A Intestinal ATAC-seq Homer Motif p-value

Supplementary Fig. 1

\begin{tabular}{lcc}
\hline & CDX2 & HNF4A/G \\
\hline & CCCATAAA & CAAAGTCCC \\
E11.5 & $1 \mathrm{e}-1320$ & - \\
E14.5 & $1 \mathrm{e}-1198$ & $1 \mathrm{e}-245$ \\
E16.5 & $1 \mathrm{e}-754$ & $1 \mathrm{e}-1606$ \\
ADVil & $1 \mathrm{e}-295$ & $1 \mathrm{e}-1418$ \\
\hline
\end{tabular}

B

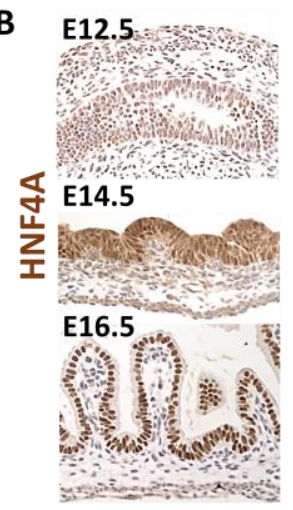

C

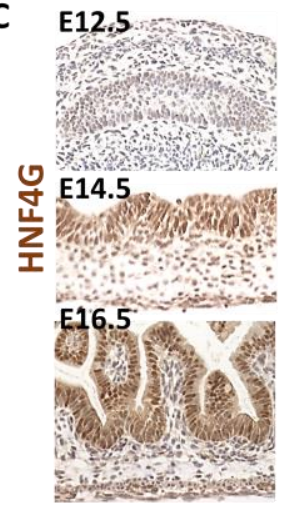

Adult

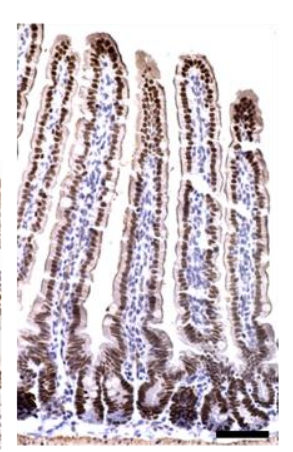

Adult

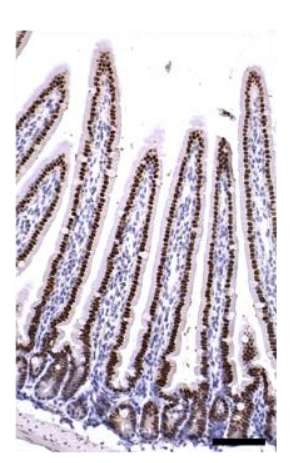

E

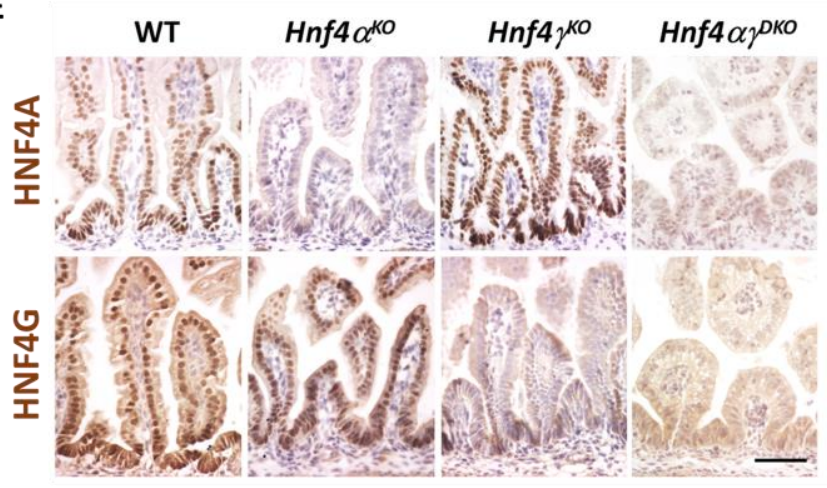

$\mathbf{F}$

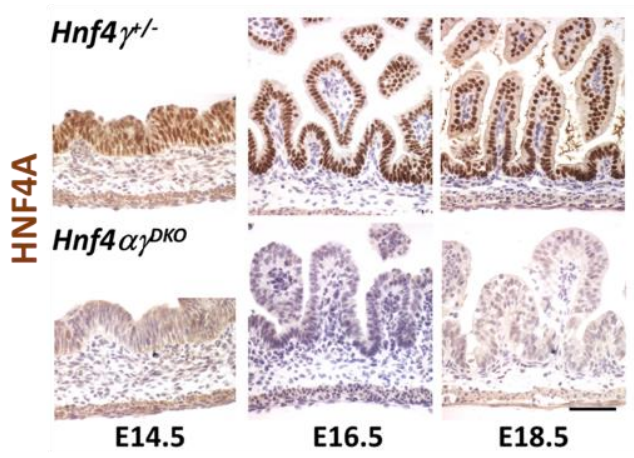

D

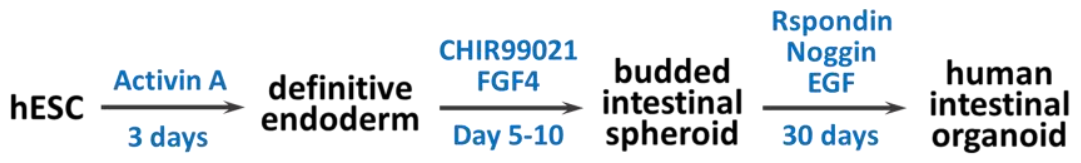

Fig. S1. HNF4 expression in the intestinal epithelium across developmental time. (A) HOMER de novo analysis of ATAC-seq (GSE115541, $\mathrm{n}=2$ biological replicates per timepoint, MACS $P$ value $\leq 10^{-5}$ ) of mouse intestinal epithelial cells at each developmental time shows that CDX2 binding motifs are present as early as E11.5, whereas HNF4 binding motifs are not present until E14.5. HNF4 motifs are increasingly abundant at accessible regions as the intestine matures. Immunostaining of (B) HNF4A and (C) HNF4G shows that relative protein levels of these factors increase with developmental time in mouse (representative of 4 biological replicates, duodenum). (D) Schematic of human intestinal organoids differentiated from hESCs (Tsai et al., 2017). (E) HNF4A and HNF4G immunostaining shows loss of HNF4 in the E18.5 intestinal epithelium of both single and double mutants (representative of 4 biological replicates, E18.5 duodenum). (F) Immunostaining of HNF4A shows loss of HNF4A in the intestinal epithelium of E14.5 Shh-Cre embryos (representative of 4 biological replicates, duodenum). Scale bars, $50 \mu \mathrm{m}$. 
A

B

C
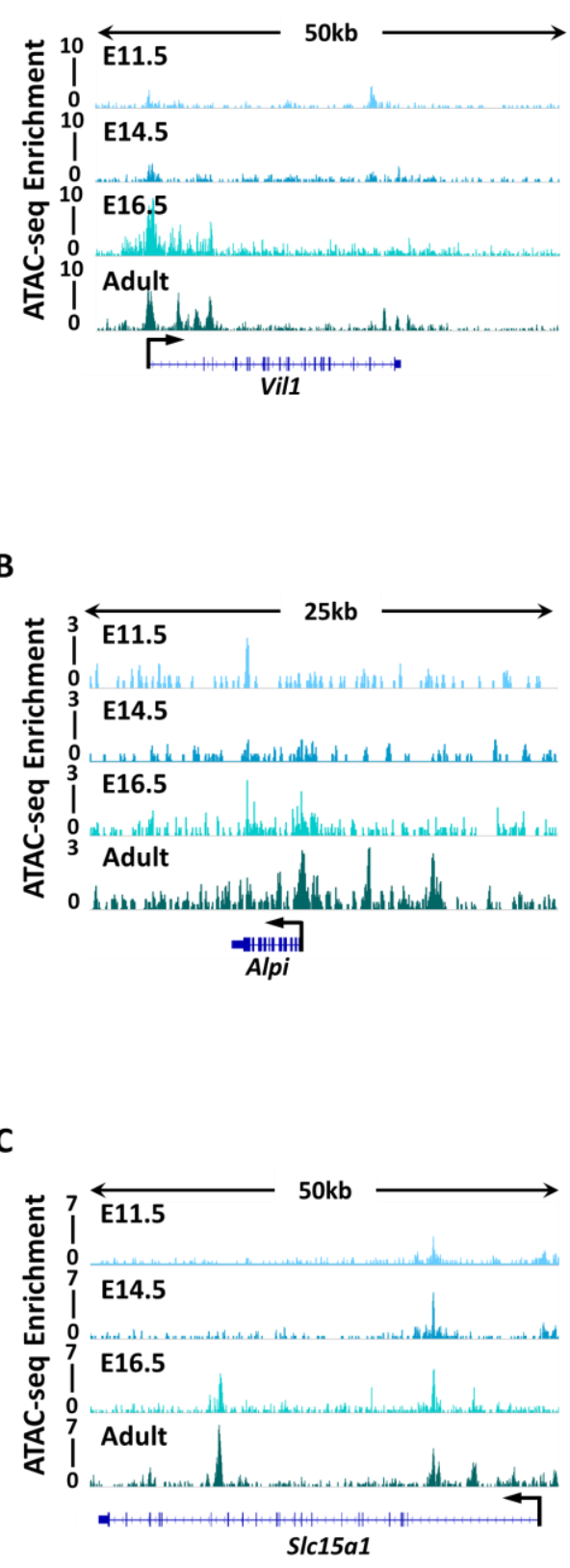

Fig. S2. HNF4 paralogs bind to and activate maturation-specific genes. Maturation-specific genes, such as (A) Vil1, (B) Alpi and (C) Slc5a1, show more accessible chromatin (ATAC-seq, left panels) and increased transcript levels (RNA-seq, right top panels) across developmental time, and HNF4 factors bind to the maturation-specific genes in the mature tissue of the adult (ChIPseq, right bottom panels). $n=2$ biological replicates per developmental timepoint for ATAC-seq

602
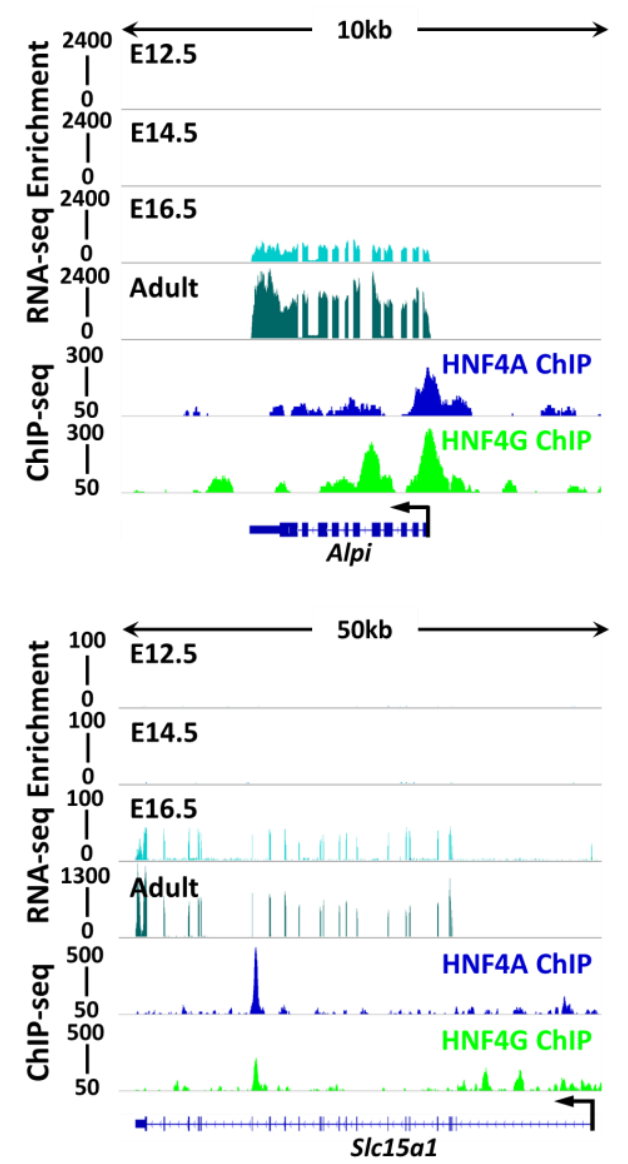
(GSE115541), RNA-seq (GSE115541) and ChIP-seq (GSE112946). 
A

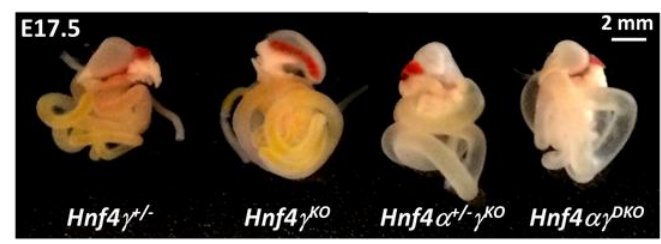

Supplementary Fig. 3

B
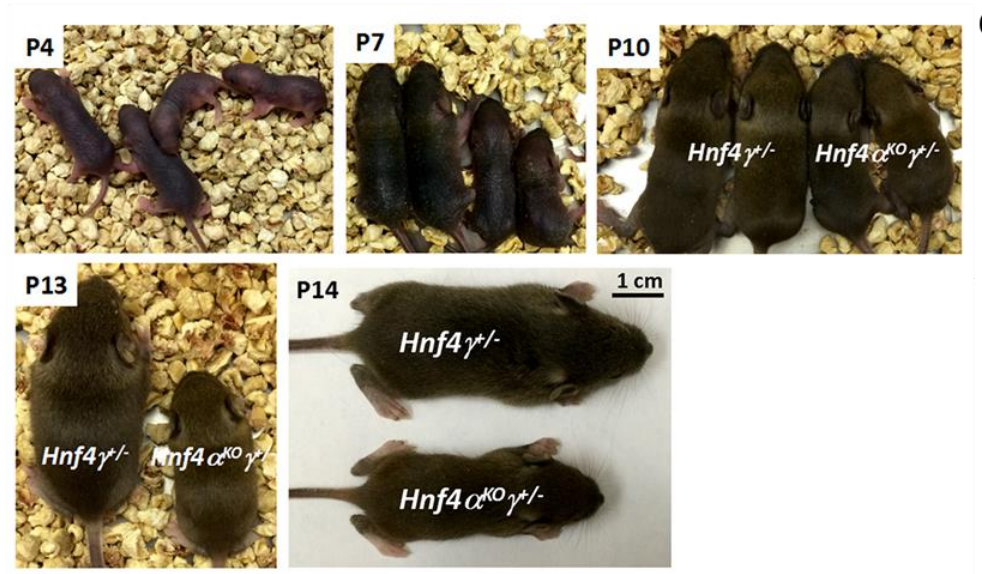

C

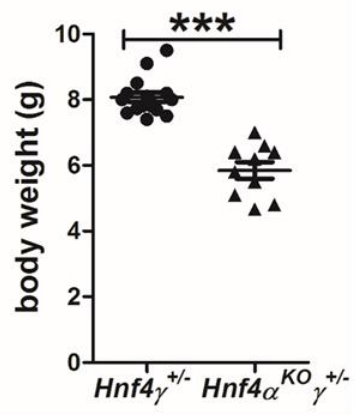

D

Fig. S3. Loss of $3 \mathrm{Hnf4}$ alleles in the developing gut leads to growth retardation after birth. (A) Loss of 3 or $4 \mathrm{Hnf} 4$ alleles in developing embryos leads to an underdeveloped intestine with distended and translucent lumen (representative of 4 biological replicates). (B) $H n f 4 \alpha^{K O} \gamma^{+/}$pups can survive after birth but show slower growth when compared to littermate controls. (C) Body weight of P14 pups. Data are presented as mean \pm SEM (Hnf4 $\gamma^{+/-}$controls: $\mathrm{n}=16 ; H n f 4 \alpha^{K O} \gamma^{+-}$ mutants: $\mathrm{n}=10$; Student's t-test, two-sided at $\mathrm{P}<0.001^{* * *}$ ). (D) Hnf4 single mutants have a similar morphology to the controls, but loss of 3 or $4 \mathrm{Hnf} 4$ alleles in developing embryos leads to strikingly stunted villi, as evidenced by H\&E staining (representative of 4 biological replicates, E18.5 duodenum; expanded panel from Fig.6H). Scale bars, $50 \mu \mathrm{m}$. 
A

$\mathrm{Hnf4} \gamma^{+/-}$

Hnf4 $\alpha \gamma^{\text {PKO }}$

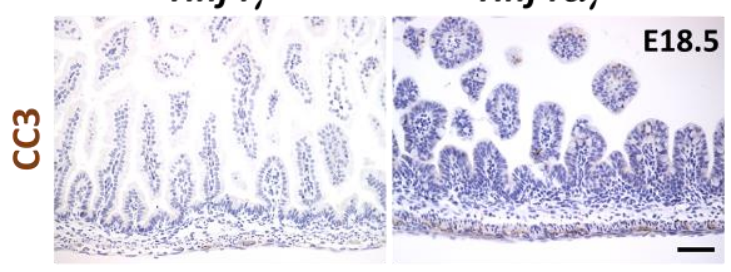

B

WT $\quad H n f 4 \alpha^{K O}$

$\mathrm{Hnf4} \gamma^{K O}$

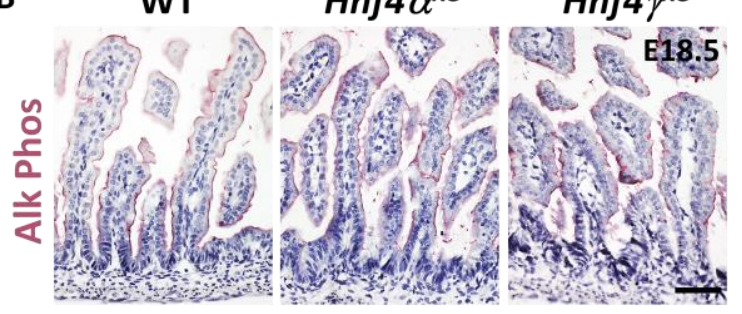

C

$\mathrm{Hnf4} \gamma^{+/-}$

Hnf4 $\alpha \gamma^{\text {PKO }}$

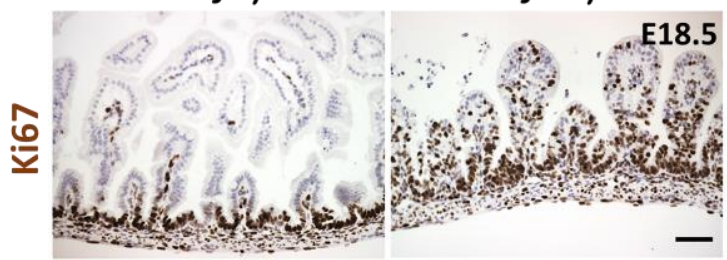

615 Fig. S4. Additional histological and immunochemical features of HNF4 mutant intestines. (A)

616 HNF4 mutants do not have significant cell death as shown in immunostaining of cleaved caspase

6173 (representative of 4 biological replicates, E18.5 duodenum). (B) $H n f 4 \alpha^{K O}$ and $H n f 4 \gamma^{K O}$ embryos

618 do not have compromised intestinal maturation, as evidenced by alkaline phosphatase staining,

619 indicating redundant roles for HNF4 factors in intestinal development (representative of 4

620 biological replicates, E18.5 duodenum). (C) Proliferative cells (Ki67 ${ }^{+}$) are observed in the villi of

$621 H n f 4 \alpha{ }^{P K O}$, which may be due to compromised tissue maturation (representative of 4 biological

622 replicates, E18.5 duodenum). Scale bars, $50 \mu \mathrm{m}$. 


\section{References}

Adachi, K., Nikaido, I., Ohta, H., Ohtsuka, S., Ura, H., Kadota, M., Wakayama, T., Ueda, H. R. and Niwa, H. (2013). Context-dependent wiring of Sox2 regulatory networks for selfrenewal of embryonic and trophoblast stem cells. Molecular cell 52, 380-392.

Babeu, J. P., Darsigny, M., Lussier, C. R. and Boudreau, F. (2009). Hepatocyte nuclear factor 4alpha contributes to an intestinal epithelial phenotype in vitro and plays a partial role in mouse intestinal epithelium differentiation. American journal of physiology. Gastrointestinal and liver physiology 297, G124-134.

Babicki, S., Arndt, D., Marcu, A., Liang, Y., Grant, J. R., Maciejewski, A. and Wishart, D. S. (2016). Heatmapper: web-enabled heat mapping for all. Nucleic acids research $\mathbf{4 4}$, W147-153.

Banerjee, K. K., Saxena, M., Kumar, N., Chen, L., Cavazza, A., Toke, N. H., O'Neill, N. K., Madha, S., Jadhav, U., Verzi, M. P., et al. (2018). Enhancer, transcriptional, and cell fate plasticity precedes intestinal determination during endoderm development. Genes \& development 32, 1430-1442.

Boyd, M., Bressendorff, S., Moller, J., Olsen, J. and Troelsen, J. T. (2009). Mapping of HNF4alpha target genes in intestinal epithelial cells. BMC gastroenterology 9, 68.

Bray, N. L., Pimentel, H., Melsted, P. and Pachter, L. (2016). Near-optimal probabilistic RNA-seq quantification. Nature biotechnology 34, 525-527.

Buenrostro, J. D., Giresi, P. G., Zaba, L. C., Chang, H. Y. and Greenleaf, W. J. (2013). Transposition of native chromatin for fast and sensitive epigenomic profiling of open chromatin, DNA-binding proteins and nucleosome position. Nature methods 10, 12131218.

Buenrostro, J. D., Wu, B., Chang, H. Y. and Greenleaf, W. J. (2015). ATAC-seq: A Method for Assaying Chromatin Accessibility Genome-Wide. Current protocols in molecular biology 109, 2129 21-29.

Cao, J., Spielmann, M., Qiu, X., Huang, X., Ibrahim, D. M., Hill, A. J., Zhang, F., Mundlos, S., Christiansen, L., Steemers, F. J., et al. (2019). The single-cell transcriptional landscape of mammalian organogenesis. Nature 566, 496-502.

Cattin, A. L., Le Beyec, J., Barreau, F., Saint-Just, S., Houllier, A., Gonzalez, F. J., Robine, S., Pincon-Raymond, M., Cardot, P., Lacasa, M., et al. (2009). Hepatocyte nuclear factor 4alpha, a key factor for homeostasis, cell architecture, and barrier function of the adult intestinal epithelium. Molecular and cellular biology 29, 6294-6308.

Chahar, S., Gandhi, V., Yu, S., Desai, K., Cowper-Sal-lari, R., Kim, Y., Perekatt, A. O., Kumar, N., Thackray, J. K., Musolf, A., et al. (2014). Chromatin profiling reveals regulatory network shifts and a protective role for hepatocyte nuclear factor 4alpha during colitis. Molecular and cellular biology 34, 3291-3304.

Chen, L., Toke, N. H., Luo, S., Vasoya, R. P., Fullem, R. L., Parthasarathy, A., Perekatt, A. O. and Verzi, M. P. (2019). A reinforcing HNF4-SMAD4 feed-forward module stabilizes enterocyte identity. Nature genetics, 10.1038/s41588-41019-40384-41580.

Chen, W. S., Manova, K., Weinstein, D. C., Duncan, S. A., Plump, A. S., Prezioso, V. R., Bachvarova, R. F. and Darnell, J. E., Jr. (1994). Disruption of the HNF-4 gene, expressed in visceral endoderm, leads to cell death in embryonic ectoderm and impaired gastrulation of mouse embryos. Genes \& development 8, 2466-2477.

Chin, A. M., Hill, D. R., Aurora, M. and Spence, J. R. (2017). Morphogenesis and maturation of the embryonic and postnatal intestine. Seminars in cell \& developmental biology 66, 8193. 
671

672

673

674

675

676

677

678

679

680

681

682

683

684

685

686

687

Consortium, E. P. (2012). An integrated encyclopedia of DNA elements in the human genome. Nature 489, 57-74.

Davison, J. M., Lickwar, C. R., Song, L., Breton, G., Crawford, G. E. and Rawls, J. F. (2017). Microbiota regulate intestinal epithelial gene expression by suppressing the transcription factor Hepatocyte nuclear factor 4 alpha. Genome research 27, 1195-1206.

Donaghey, J., Thakurela, S., Charlton, J., Chen, J. S., Smith, Z. D., Gu, H., Pop, R., Clement, K., Stamenova, E. K., Karnik, R., et al. (2018). Genetic determinants and epigenetic effects of pioneer-factor occupancy. Nature genetics 50, 250-258.

Duncan, S. A., Nagy, A. and Chan, W. (1997). Murine gastrulation requires HNF-4 regulated gene expression in the visceral endoderm: tetraploid rescue of $\mathrm{Hnf}-4(-/-)$ embryos. Development 124, 279-287.

Gao, N., White, P. and Kaestner, K. H. (2009). Establishment of intestinal identity and epithelialmesenchymal signaling by Cdx2. Developmental cell 16, 588-599.

Garrison, W. D., Battle, M. A., Yang, C., Kaestner, K. H., Sladek, F. M. and Duncan, S. A. (2006). Hepatocyte nuclear factor 4alpha is essential for embryonic development of the mouse colon. Gastroenterology 130, 1207-1220.

Grainger, S., Savory, J. G. and Lohnes, D. (2010). Cdx2 regulates patterning of the intestinal epithelium. Developmental biology 339, 155-165.

Harfe, B. D., Scherz, P. J., Nissim, S., Tian, H., McMahon, A. P. and Tabin, C. J. (2004). Evidence for an expansion-based temporal Shh gradient in specifying vertebrate digit identities. Cell 118, 517-528.

Harper, J., Mould, A., Andrews, R. M., Bikoff, E. K. and Robertson, E. J. (2011). The transcriptional repressor Blimp1/Prdm1 regulates postnatal reprogramming of intestinal enterocytes. Proceedings of the National Academy of Sciences of the United States of America 108, 10585-10590.

Hayhurst, G. P., Lee, Y. H., Lambert, G., Ward, J. M. and Gonzalez, F. J. (2001). Hepatocyte nuclear factor 4alpha (nuclear receptor 2A1) is essential for maintenance of hepatic gene expression and lipid homeostasis. Molecular and cellular biology 21, 1393-1403.

Heinz, S., Benner, C., Spann, N., Bertolino, E., Lin, Y. C., Laslo, P., Cheng, J. X., Murre, C., Singh, H. and Glass, C. K. (2010). Simple combinations of lineage-determining transcription factors prime cis-regulatory elements required for macrophage and B cell identities. Molecular cell 38, 576-589.

Huang da, W., Sherman, B. T. and Lempicki, R. A. (2009). Systematic and integrative analysis of large gene lists using DAVID bioinformatics resources. Nature protocols 4, 44-57.

Kazakevych, J., Sayols, S., Messner, B., Krienke, C. and Soshnikova, N. (2017). Dynamic changes in chromatin states during specification and differentiation of adult intestinal stem cells. Nucleic acids research 45, 5770-5784.

Kim, D., Pertea, G., Trapnell, C., Pimentel, H., Kelley, R. and Salzberg, S. L. (2013). TopHat2: accurate alignment of transcriptomes in the presence of insertions, deletions and gene fusions. Genome biology 14, R36.

Kumar, N., Tsai, Y. H., Chen, L., Zhou, A., Banerjee, K. K., Saxena, M., Huang, S., Toke, N. H., Xing, J., Shivdasani, R. A., et al. (2019). The lineage-specific transcription factor CDX2 navigates dynamic chromatin to control distinct stages of intestine development. Development 146.

Langmead, B. and Salzberg, S. L. (2012). Fast gapped-read alignment with Bowtie 2. Nature methods 9, 357-359.

Li, J., Ning, G. and Duncan, S. A. (2000). Mammalian hepatocyte differentiation requires the transcription factor HNF-4alpha. Genes \& development 14, 464-474. 
Lindeboom, R. G., van Voorthuijsen, L., Oost, K. C., Rodriguez-Colman, M. J., Luna-Velez, M. V., Furlan, C., Baraille, F., Jansen, P. W., Ribeiro, A., Burgering, B. M., et al. (2018). Integrative multi-omics analysis of intestinal organoid differentiation. Molecular systems biology 14, e8227.

Liu, T., Ortiz, J. A., Taing, L., Meyer, C. A., Lee, B., Zhang, Y., Shin, H., Wong, S. S., Ma, J., Lei, Y., et al. (2011). Cistrome: an integrative platform for transcriptional regulation studies. Genome biology 12, R83.

Love, M. I., Huber, W. and Anders, S. (2014). Moderated estimation of fold change and dispersion for RNA-seq data with DESeq2. Genome biology 15, 550.

Madison, B. B., Dunbar, L., Qiao, X. T., Braunstein, K., Braunstein, E. and Gumucio, D. L. (2002). Cis elements of the villin gene control expression in restricted domains of the vertical (crypt) and horizontal (duodenum, cecum) axes of the intestine. The Journal of biological chemistry 277, 33275-33283.

McLean, C. Y., Bristor, D., Hiller, M., Clarke, S. L., Schaar, B. T., Lowe, C. B., Wenger, A. M. and Bejerano, G. (2010). GREAT improves functional interpretation of cis-regulatory regions. Nature biotechnology 28, 495-501.

Mould, A. W., Morgan, M. A., Nelson, A. C., Bikoff, E. K. and Robertson, E. J. (2015). Blimp1/Prdm1 Functions in Opposition to Irf1 to Maintain Neonatal Tolerance during Postnatal Intestinal Maturation. PLoS genetics 11, e1005375.

Nerurkar, N. L., Mahadevan, L. and Tabin, C. J. (2017). BMP signaling controls buckling forces to modulate looping morphogenesis of the gut. Proceedings of the National Academy of Sciences of the United States of America 114, 2277-2282.

Niwa, H. (2018). The principles that govern transcription factor network functions in stem cells. Development 145.

Nord, A. S., Blow, M. J., Attanasio, C., Akiyama, J. A., Holt, A., Hosseini, R., Phouanenavong, S., Plajzer-Frick, I., Shoukry, M., Afzal, V., et al. (2013). Rapid and pervasive changes in genome-wide enhancer usage during mammalian development. Cell 155, 1521-1531.

Parviz, F., Matullo, C., Garrison, W. D., Savatski, L., Adamson, J. W., Ning, G., Kaestner, K. H., Rossi, J. M., Zaret, K. S. and Duncan, S. A. (2003). Hepatocyte nuclear factor 4alpha controls the development of a hepatic epithelium and liver morphogenesis. Nature genetics 34, 292-296.

Pinello, L., Farouni, R. and Yuan, G. C. (2018). Haystack: systematic analysis of the variation of epigenetic states and cell-type specific regulatory elements. Bioinformatics 34, 19301933.

Quinlan, A. R. (2014). BEDTools: The Swiss-Army Tool for Genome Feature Analysis. Current protocols in bioinformatics 47, 1112 11-34.

Ramirez, F., Ryan, D. P., Gruning, B., Bhardwaj, V., Kilpert, F., Richter, A. S., Heyne, S., Dundar, F. and Manke, T. (2016). deepTools2: a next generation web server for deep-sequencing data analysis. Nucleic acids research 44, W160-165.

Robinson, J. T., Thorvaldsdottir, H., Winckler, W., Guttman, M., Lander, E. S., Getz, G. and Mesirov, J. P. (2011). Integrative genomics viewer. Nature biotechnology 29, 24-26.

Sekiya, T., Muthurajan, U. M., Luger, K., Tulin, A. V. and Zaret, K. S. (2009). Nucleosomebinding affinity as a primary determinant of the nuclear mobility of the pioneer transcription factor FoxA. Genes \& development 23, 804-809.

Shen, Y., Yue, F., McCleary, D. F., Ye, Z., Edsall, L., Kuan, S., Wagner, U., Dixon, J., Lee, L., Lobanenkov, V. V., et al. (2012). A map of the cis-regulatory sequences in the mouse genome. Nature 488, 116-120. 
Shin, H., Liu, T., Manrai, A. K. and Liu, X. S. (2009). CEAS: cis-regulatory element annotation system. Bioinformatics 25, 2605-2606.

Soneson, C., Love, M. I. and Robinson, M. D. (2015). Differential analyses for RNA-seq: transcript-level estimates improve gene-level inferences. F1000Research 4, 1521.

Tsai, Y. H., Nattiv, R., Dedhia, P. H., Nagy, M. S., Chin, A. M., Thomson, M., Klein, O. D. and Spence, J. R. (2017). In vitro patterning of pluripotent stem cell-derived intestine recapitulates in vivo human development. Development 144, 1045-1055.

Verzi, M. P., Shin, H., He, H. H., Sulahian, R., Meyer, C. A., Montgomery, R. K., Fleet, J. C., Brown, M., Liu, X. S. and Shivdasani, R. A. (2010). Differentiation-specific histone modifications reveal dynamic chromatin interactions and partners for the intestinal transcription factor CDX2. Developmental cell 19, 713-726.

Verzi, M. P., Shin, H., Ho, L. L., Liu, X. S. and Shivdasani, R. A. (2011). Essential and redundant functions of caudal family proteins in activating adult intestinal genes. Molecular and cellular biology 31, 2026-2039.

Verzi, M. P., Shin, H., San Roman, A. K., Liu, X. S. and Shivdasani, R. A. (2013). Intestinal master transcription factor CDX2 controls chromatin access for partner transcription factor binding. Molecular and cellular biology 33, 281-292.

Visel, A., Blow, M. J., Li, Z., Zhang, T., Akiyama, J. A., Holt, A., Plajzer-Frick, I., Shoukry, M., Wright, C., Chen, F., et al. (2009). ChIP-seq accurately predicts tissue-specific activity of enhancers. Nature 457, 854-858.

Walton, K. D., Freddo, A. M., Wang, S. and Gumucio, D. L. (2016a). Generation of intestinal surface: an absorbing tale. Development 143, 2261-2272.

Walton, K. D., Kolterud, A., Czerwinski, M. J., Bell, M. J., Prakash, A., Kushwaha, J., Grosse, A. S., Schnell, S. and Gumucio, D. L. (2012). Hedgehog-responsive mesenchymal clusters direct patterning and emergence of intestinal villi. Proceedings of the National Academy of Sciences of the United States of America 109, 15817-15822.

Walton, K. D., Whidden, M., Kolterud, A., Shoffner, S. K., Czerwinski, M. J., Kushwaha, J., Parmar, N., Chandhrasekhar, D., Freddo, A. M., Schnell, S., et al. (2016b). Villification in the mouse: Bmp signals control intestinal villus patterning. Development 143, 427-436.

Wells, J. M. and Spence, J. R. (2014). How to make an intestine. Development 141, 752-760.

Wilkinson, A. C., Nakauchi, H. and Gottgens, B. (2017). Mammalian Transcription Factor Networks: Recent Advances in Interrogating Biological Complexity. Cell systems 5, 319331.

Zhang, Y., Liu, T., Meyer, C. A., Eeckhoute, J., Johnson, D. S., Bernstein, B. E., Nusbaum, C., Myers, R. M., Brown, M., Li, W., et al. (2008). Model-based analysis of ChIP-Seq (MACS). Genome biology 9, R137. 